\title{
ArchiRev-Reverse engineering of information systems toward ArchiMate models. An industrial case study
}

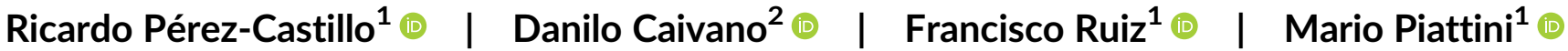

\author{
${ }^{1}$ Information Technology \& Systems Institute \\ (ITSI), University of Castilla-La Mancha, Ciudad \\ Real, Spain \\ ${ }^{2}$ Department of Informatics, University of Bari, \\ Bari, Italy \\ Correspondence \\ Ricardo Pérez-Castillo, Information \\ Technology \& Systems Institute (ITSI), \\ University of Castilla-La Mancha, Paseo de la \\ Universidad 4 13071, Ciudad Real, Spain. \\ Email: ricardo.pdelcastillo@uclm.es \\ Funding information \\ Junta de Comunidades de Castilla-La Mancha, \\ Grant/Award Numbers: G3SOFT \\ (SBPLY/17/180501/000150), GEMA \\ (SBPLY/17/180501/000293), SOS \\ (SBPLY/17/180501/000364); Ministerio de \\ Economía, Industria y Competitividad, \\ Gobierno de España, Grant/Award Numbers: \\ BIZDEVOPS-Global (RTI2018-098309-B- \\ C31), ECLIPSE (RTI2018-094283-B-C31) \\ Ministerio de Economía, Industría y \\ Competitividad y Fondos FEDER
}

\begin{abstract}
In the context of digital transformation, companies are increasingly forced to develop, manage and continually align IT and business. Companies have found in Enterprise Architecture (EA) a valuable tool to represent and manage IT and business in a holistic way by establishing connections among technology concerns and business/strategical/motivational ones. EA modelling is one of the most critical tasks in this context to represent accurate models that make the difference in the decision-making and governance processes of the company. In most of the cases, EA models are manually defined by experts which is usually error-prone and time-consuming. Thus, the constant business and IT realignment through manual modelling becomes complex and expensive. This research presents ArchiRev, an extensible reverse engineering method that automate the extraction of EA models (using ArchiMate language) by analysing different information systems artefacts. This proposal has been validated through an industrial case study in the context of a C\#-based system of an Italian ship refurbishment company. The study has demonstrated the effectiveness (based in the opinion of some business experts) and efficiency of the method. The main implication is that EA modelling can be accelerated, whereas subjectivity and errors introduced by experts might be reduced.

KEYWORDS

ArchiMate, ArchiRev, BizDevOps, enterprise architecture, reverse engineering, TOGAF
\end{abstract}

\section{1 | INTRODUCTION}

Digital transformation is changing productive models and how companies operate in daily basis for offering disruptive products and services. ${ }^{1} \mathrm{~A}$ cornerstone for digital transformation is the best alignment of business and Information Technologies (IT). ${ }^{2}$ However, the operative alignment between business and IT is hardly ever achieved and, when it is achieved, the necessary agile, continuous adaptation is not trivial in a world with changing markets and volatile IT. ${ }^{3}$ Consequently, there is a recent trend of companies towards the BizDevOps paradigm (see Figure 1 ), in which companies continuously (re-)define business goals and processes as well as the respective functionality of their (micro)services, (re)developing and operating them. ${ }^{5}$ In the mentioned context, Enterprise Architecture (EA) deserves special mention because it has demonstrated to be a useful tool for managing business and IT perspectives in an integrated and holistic manner according to the mentioned BizDevOps paradigm, which favours the mentioned alignment between business and IT. ${ }^{6}$ As a result, EA can be a catalyst for digital transformation and it have been proven it is extremely useful for making decisions in this regard. ${ }^{7}$

Today, most companies are conscious of constantly reengineering their business cores and processes to respond proactively to changing market demands and technological evolution. ${ }^{1,8}$ More and more companies are perceiving that EA is a mechanism that allow them to handle system engineering and business changes supported by such systems. ${ }^{9}$ 


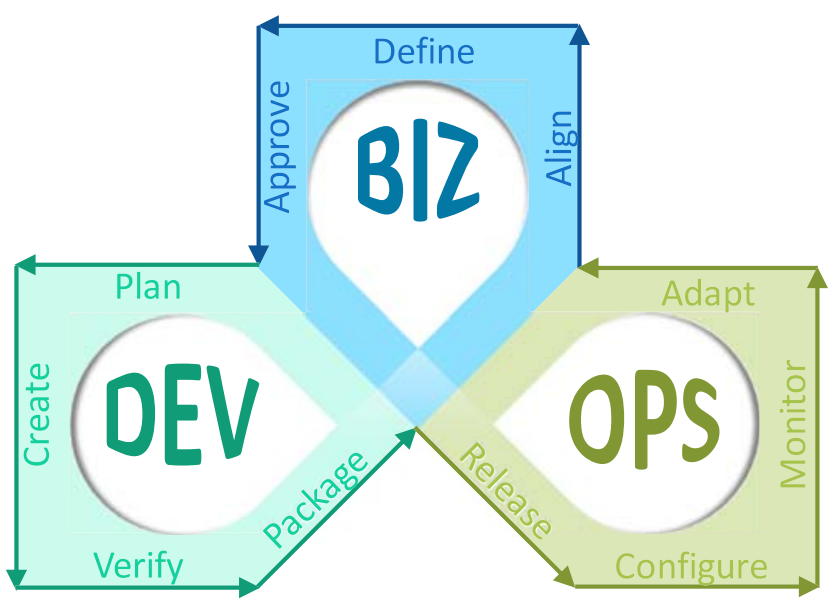

FIGURE 1 BizDevOps paradigm with three integrated cycles: business (Biz), development (Dev) and operations (Ops) (adapted from Putano ${ }^{4}$ )

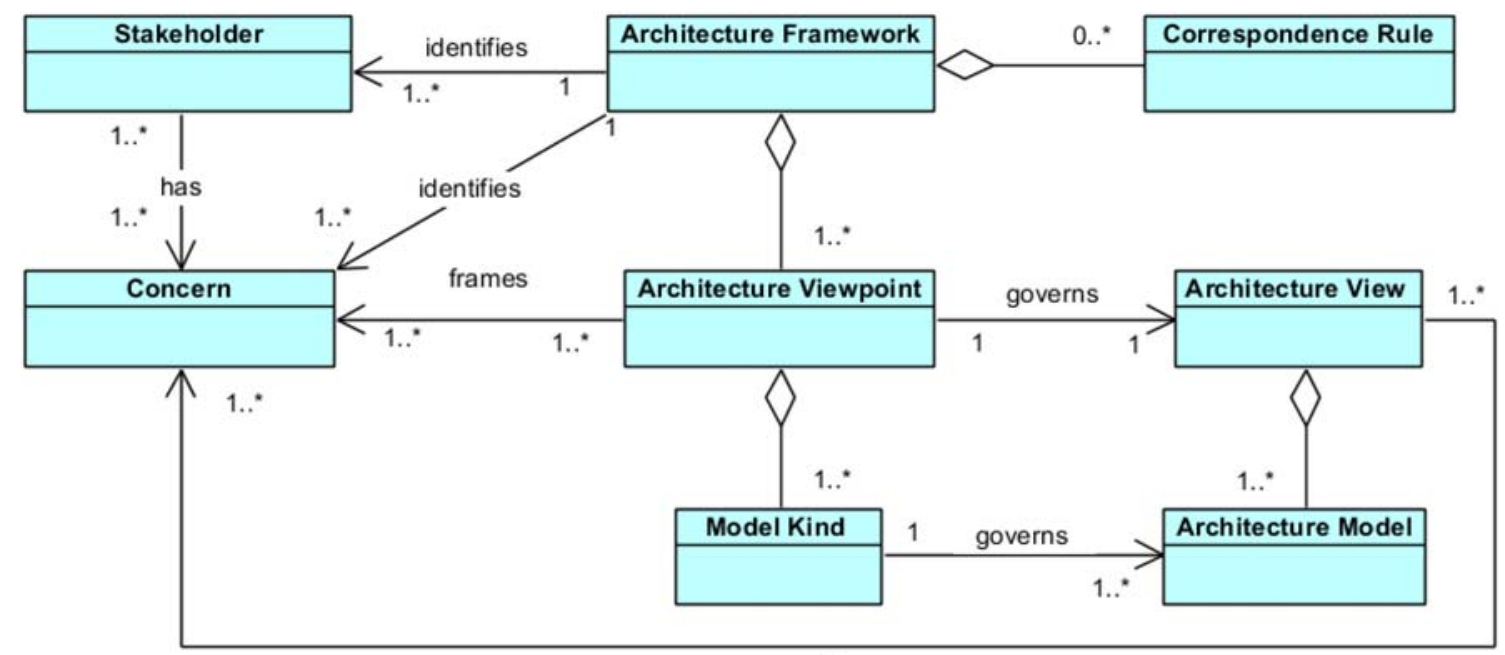

addresses

FIGURE 2 Constructs of any architecture framework according to ISO/IEC/IEEE 42010

In order to accomplish Enterprise Architecture Management (EAM), ${ }^{6}$ one of the most important issues is creating EA models that represents different viewpoints of a company for managing its different concerns (e.g., processes, services, applications, data and infrastructure) that accurately represents the realm of such a company and its IT landscape. In an EAM context, there are at least two main purposes to create enterprise models. ${ }^{10}$ First, EA models can be used to describe the baseline/current situation of the enterprise. Second, EA models can be also employed to describe the (desired) target/future situation. Enterprise cartography ${ }^{11}$ provides an understanding of the dynamics of an organization through its EA models, that is, as-was (past), as-is (present) and to-be (future).

Most traditional EAM approaches usually consider manual modelling from scratch that is carried out by business and IT experts aided by EAM suites. Although different sources of knowledge within the company might be often used for modelling such EA models, EA modelling is still a main responsibility of experts with a low degree of automation. ${ }^{12,13}$ In most of the cases, the knowledge sources for modelling EA are the own organization's employees. These people deliver the information through experts' interviews and documentation and manuals regarding processes, rules, responsibilities and structures in the organization and other technical documentations regarding underlaying IT infrastructure. IS are, however, alternative, powerful sources of knowledge to achieve the automation of EA modelling. IS usually provide information related to both behaviour and structure of EA (see Figure 3). This information might be extracted by analysing some of these IS artefacts such as source code, data models and service configuration files. ${ }^{15}$ Many authors consider IS as one of the most valuable assets and most effective enablers for modelling EA. ${ }^{16}$ This is owing to the fact that these systems gather information providing access and coordination to such information throughout the whole organization. Actually, many times IS are the main source of implicit knowledge of the organization.

Having modelled EA from IS, it should be noticed that EA models are not static artefacts within companies; instead, EA models need to evolve over time in order to consider the continuous, agile changes in the existing architecture and business according to new approaches like BizDevOps. ${ }^{17}$ Many times, the practically continuous update of EA models generates conflicts as a result of the introduction of new IS whereas 
FIGURE 3 ArchiMate 3 core framework (adapted from The Open Group ${ }^{14}$ )

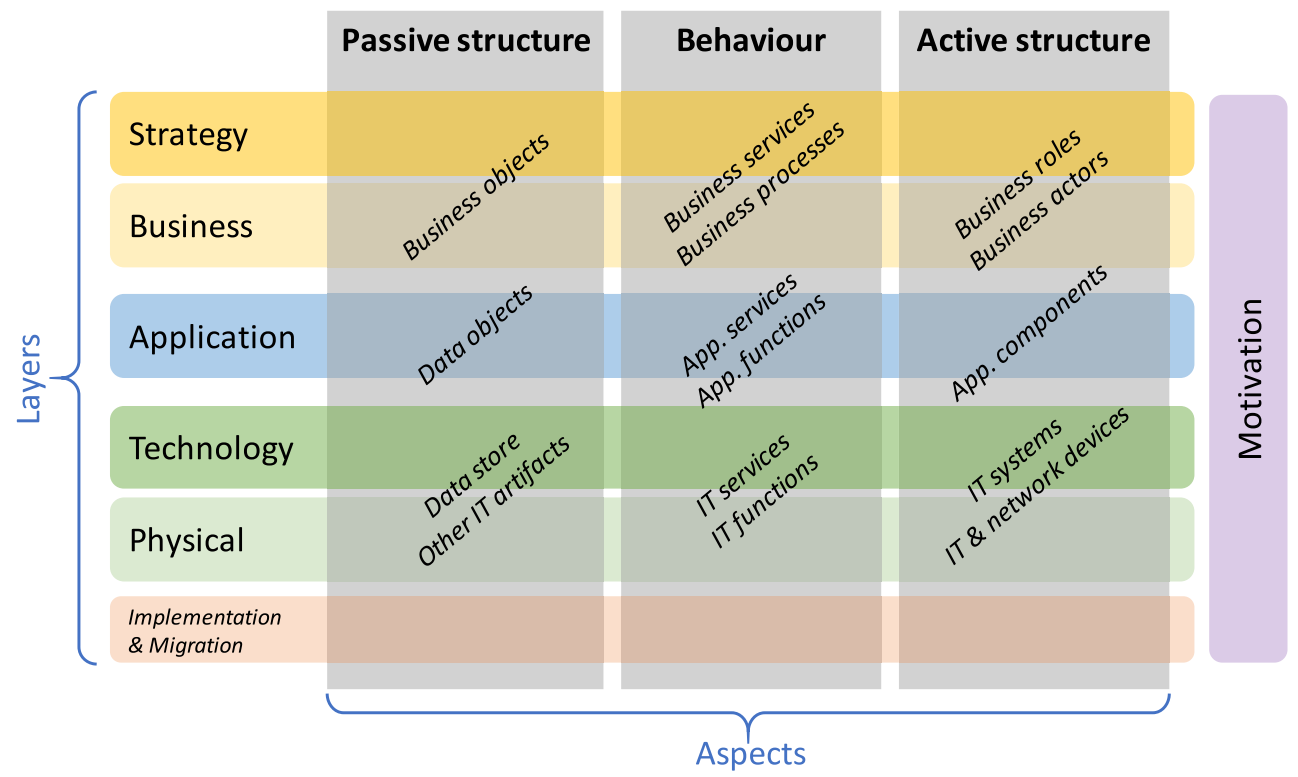

business operations have to continue in parallel. ${ }^{18}$ Actually, the uncontrolled proliferation in the IT infrastructure landscape can lead to the need of update EA models in shorter periods of time. What is more, many companies employ EA modelling for sanitizing their complex IT landscapes. ${ }^{19}$

According to Farwick, ${ }^{20}$ a critical need for EA modelling and maintenance is to decrease the amount of manual work and automate it up to a certain extent in order to gain accuracy and consistency. Manual EA modelling attains the following flaws ${ }^{21}$ :

- Error-proneness: Manual modelling can lead to EA models with missing elements (i.e., false negatives) and irrelevant elements (i.e., false positives). In other words, different experts modelling EA models for the same company could deliver EA models that differ in a greater extent. Additionally, many companies use inadequate EA management tools, for example, some general-purpose tools like text processors, spreadsheets, or unstructured diagrams. ${ }^{15}$

- Time-consuming: As any manual task that is not automated, manual modelling incurs in additional time to delivers EA models. First, business experts usually deal with blank page syndrome that contributes to delays in many cases. Second, because it is an error-prone task, error fixing consumes extra time.

- Slow and poor re-adaptation: EA models try to integrate different viewpoints of the company in order to align business and IT. In real-world companies, business can change so frequent as IT infrastructure does. As a result, EA models must be changed and re-adapted to represent the EA of a company in an accurate way. Otherwise, company could manage outdated models that are useless, and in the worst case, the usage of this inaccurate models could lead to make wrong decisions with potential harmful effects for the company. ${ }^{13}$

- Expensive: On the one hand, manual EA modelling is expensive mainly because EA modelling is typically a human-intensive job and it often leads to conciliations in terms of the amount of resources assigned in EA modelling projects that is directly related to the quality of EA models. Additional expenses are associated with the time-consuming nature and also with the error fixing and re-adaptations that are continuously needed to avoid outdated models that do not accurately represent the enterprise. ${ }^{12}$ Even more, these EA architects and related profiles are scarce and, therefore, expensive. On the other hand, EA models that are used to express the desired, future situation of the enterprise also need continuous re-adaptations because of changing business goals and volatile technology landscapes. ${ }^{10}$

Because of all these flaws, some new approaches attempting to automate EA modelling have come up for dealing with the mentioned flaws. ${ }^{22-25}$ Automatic EA modelling needs less time and, therefore, is cheaper than manual modelling. As a result, EA models are adapted and re-aligned with IT faster, because these models can be continuously reextracted in an easy way. Additionally, in comparison with manual EA modelling by experts, these methods are less error-prone and minimize the subjectivity of the analysis. Unfortunately, automatic EA modelling must address some open issues. For example, IS are provided by third-party companies, signifying that IS descriptions could be uncertain, thus leading to incomplete and inaccurate EA models. Also, these automated methods are based on reverse engineering techniques, which can lead to semantic losses in the outgoing EA models. Apart from this, the main limitation of such techniques is that the most abstract EA information (such as motivational aspects and business concerns) is almost impossible to be extracted from IS that are at the medium and low level of abstraction. However, the usage of automated techniques for EA modelling can be used in combination with some manual work done by business experts. ${ }^{21}$

Despite existing proposals, this research area is not fully mature yet. ${ }^{26}$ The automation of EA modelling has followed a big bang approach in last years with a lot of different proposals without enough empirical evidence of their feasibility and applicability to industry. Authors 
consider a lot of different sources of knowledge related to Information Systems (IS) and IT infrastructure as input for the proposed mining and elicitation techniques. Although many of these proposals contribute to the automation of EA model extraction, the majority of the proposals are not supported by a tool in order to ensure their replicability and application in industry to a broader extent. Thereby, this paper proposes ArchiRev, an extensible reverse engineering method to automatically generate ArchiMate models by analysing different IS sources of knowledge. The main contribution of this paper is that ArchiRev is specifically tailored for analysing source code of IS and generating EA viewpoints representing the application layer. Together with this technique, a tool has been developed which has facilitated the application of the ArchiRev method. Using ArchiRev tool, an industrial case study has been conducted. The study analysed a C\# system belonging to an Italian ship refurbishment company devoted to manage and plan the ship measurement taking and material ordering. The study has involved some business experts to assess precision and recall (which have respectively reached $68 \%$ and $80 \%$ ) demonstrating the effectiveness of the method. The efficiency of the method has been also evaluated the scalability analysis, which demonstrated to be linear regarding the size of the system. The main implication of this research is that the study offers empirical evidence of the feasibility and applicability in the industry of the automation of EA modelling.

The remaining of this article is organized as follows. Section 2 presents a brief background about EA and some EA modelling frameworks. Section 3 summarizes some related works in the field of EA mining and other reverse engineering techniques to extract EA models. Section 4 presents in detail ArchiRev and its associated tool. Section 5 disseminates the case study conducted with the industrial system. Finally, Section 6 provides conclusions and future work.

\section{2 | ENTERPRISE ARCHITECTURE AND ARCHIMATE BACKGROUND}

The EA term is often confusing, and it is muddled with traditional architecture approaches such as software or IS architectures. However, traditional architecture approaches address technology aspects ${ }^{27}$ whereas EA paradigms are business-friendly rather than merely an IT approach. Thereby, EA supports an effective (usually holistic) IT governance and alignment of business and IT.

EA can provide companies with "a holistic understanding of all aspects of a business, its drivers and surrounding environments, its business processes, organizational structures, information flows, IT systems, and technical infrastructures." ${ }^{28}$ Thus, EA offers different viewpoints of an organization which are associated with different business rationale elements (mainly ends/objectives, means/courses of action and influencers/drivers). ${ }^{29}$ EA can, therefore, reduce the complexity of governance and management of organizations to "improve communication, align business and IT, and drive organizational change." ${ }^{10}$

During last years, several EA frameworks have been proposed, and some of them have gained certain adoption in the industry, for instance, TOGAF, DoDAF and MODAF, Zachman and ESARC. The majority of these frameworks are compliant with ISO/IEC/IEEE $42010^{31}$ and specially with the metamodel this standard proposes for describing architectures (see Figure 2).

Frameworks for EA are defined as compositions of various architectural viewpoints. A viewpoint concerns one (or various) stakeholder regarding one interested aspect. Viewpoint defines the expected conventions for one type of architectural view. Such conventions (in the context of architectural viewpoints) include things such as "languages, notations, model kinds, design rules, and/or modelling methods, analysis techniques and other operations on views." ${ }^{14}$ As a result, we can state that EA viewpoints are means to cope with the extreme complexity in EA management.

The TOGAF framework ${ }^{32}$ is extensively adopted in the market and can be said that it is the de facto standard. ${ }^{33,34}$ TOGAF proposes the architecture development method (ADM) as an iterative methodology for defining EA. In addition to EA frameworks, modelling languages and specifications are necessary to depict all EA concerns in different architectural viewpoints. The Open Group ${ }^{14}$ also released ArchiMate, a modelling language (aligned with TOGAF) with which to represent different EA information models. ArchiMate allows the modelling of EA from different viewpoints, in which the position within the cells highlights the stakeholders' concerns (see Figure 3).

ArchiMate proposes layers and aspects as the two main dimensions for organizing all the elements. Core layers represent the three levels at which it is possible to model an enterprise in ArchiMate, that is, business, application and technology. Aspects refers to (i) the active structure (elements representing who/what makes the things), (ii) behaviour (elements indicating what is made and how it is made) and (iii) passive structure (things on which behaviour is performed). Despite the mentioned structure, composite elements belonging to various aspects are also allowed. Finally, it should be noticed that the last ArchiMate specification (version 3) defines further layers for strategy, physical and implementation/migration elements and fourth additional aspects with motivational elements.

\section{3 | RELATED WORK}

This section collects related work by summarizing some of the main findings that authors figured out in a previous systematic mapping study we conducted. ${ }^{26}$ 
In spite of the origin of EA term comes from 80s, the research on the EAM field has been intensified in the last years. Actually, other research areas are converging into the EA field like, for example, digital transformation, (micro)service-oriented architectures (SOA), business process management, model-driven engineering (MDE), systems-of-systems engineering, between other relevant areas. EA modelling and maintenance are important aspects within the field. In particular, automatic EA modelling is showing an increasing interest in the community. Both academia and industry claim for methods and techniques that aid the manual data collection processes by means of tools. Actually, there are some (semi) automatic techniques and method for extracting EA models from various IS artefacts among other related sources of information in the literature.

Related works employ different sources of knowledge to model all the different EA elements and those works use different techniques. ${ }^{26}$ Having analysed such variation, we detected some recurrent patterns. For instance, data collection and data mapping, which consider different inputs and outputs, is the most common technique. Farwick et $\mathrm{al}^{12,20,23}$ semiautomated data collection processes from external data sources and events. These works provide a specific metamodel and draw some techniques to achieve a better synchronization between EA models and what they represent in the real world. They provide a draft of the technical architecture of a reverse engineering tool to support the proposed method. Similarly, Sánchez et al $^{15}$ provided an approach that gather "information from multiple sources such as information systems, databases, files (system's logs, source code, configuration), and previously existing models" and create enterprise models in a semi-automatic manner. This method is implemented in a tool called EM-AutoBuilder.

Other patterns detected in related work are those proposals applying process mining techniques under process logs. ${ }^{35}$ For example, Van Langerak et $\mathrm{al}^{36}$ proposed a technique to analyse runtime execution data and visualize the respective runtime enterprise architecture based on results of different process execution analysis. This technique enables the visualization of flows within the organization network and IT systems; thus, it helps to understand the execution of business operations and how IT systems interacts in such operations. Liu et al ${ }^{37}$ recover component-based architectures from software execution data. Repta et $\mathrm{al}^{25}$ propose a generic framework that help to develop "automated process mapping applications for flexible and complex processes" in the context of EA. However, this work does not demonstrate its feasibility because it does not provide a supporting tool. Truong et al ${ }^{38}$ proposed a method that combines "enterprise's strategy together with data mining rules extracted from the data warehouse of the enterprise in order to make design-time changes to its business processes." This method tries to discard redundant tasks and reorder some business process tasks inefficiently located. The idea of this realignment approach, according to the authors, is "to combine yesterday's behavioural facts (i.e., mining enterprise's operational data) with today's operationalization possibilities (e.g., goals), which results in tomorrow's business model." Werf et $\mathrm{al}^{39}$ also consider operational data for extracting architectural descriptions in which quality attributes are considered apart from functional aspects.

In a similar way and with the goal of generating EA models concerning service viewpoints, MDE approaches are also used. For example, Ge et $\mathrm{al}^{40}$ follows an MDE approach for modelling a system-of-systems (SoS) architecture framed in the DoDAF Metamodel. This work facilitates the automated transformation of executable models from architectural instance data. Following this line, Hu et al ${ }^{41}$ define an MDE method for service-oriented SoS architecting, modelling and simulation. This work employs SysML to cope with the intrinsic complexity of SoS and make it possible the alignment between IT infrastructure and high-level complex business requirements. Bogner et al ${ }^{22}$ also uses some metamodeling principles together with some ontology-based methods for "integrating Microservice Architectures (MSA) by extending original enterprise architecture reference models with elements for more flexible architectural metamodels." Also, Granchelli et al ${ }^{42}$ automatically represent MSA with a domain-specific language.

Finally, as we mentioned before, many techniques still combine some (semi)automatic processes with expert's knowledge collected through interviews or similar methods. For example, Loucopoulos et $\mathrm{al}^{16}$ provide a conceptual framework for "capability-driven development of enterprise knowledge and discuss how this can be used to enable the design of capabilities and services" of companies. This work outlines a tool that aids EA modelling as made by experts. This uses a certain notation and stablishes some automatic analyses and transformations between models. Santana et $\mathrm{al}^{43}$ defined a method combining structural information and some expert's tacit knowledge. With such information, the proposed method refines the expert's knowledge about the architecture (so-called "cognitive-structural diagnosis analysis and attribute check analysis"). These analyses allow enterprise architects to minimize analysis subjectivity while validating important components and also suggesting important structural ones to be further analysed. Similarly, Pulkkinen et al ${ }^{44}$ provides a method for establishing collaboration between different stakeholders, both from the IT and business groups, in order to improve EA modelling projects. Rosasco et al ${ }^{45}$ defined a systematic method "for eliciting required EA knowledge from stakeholders" which is based on queries that follows the approach of the Vision-Mission-Objectives-Strategy-Tactics (VMOST). This method is able to document and analyse the elicited EA requirements in a semiautomatic way.

Despite the aforementioned related work, these proposals have some limitations since those work do not fulfil the following four conditions together, ${ }^{26}$ which we believe are important and motivate ArchiRev:

- Standard compliant: Some of these proposals used ad hoc EA notations or some specifications that are not widely used in the industry as, for example, ArchiMate is. As a result, this limits the applicability of those techniques in most of the companies.

- Extensibility: Most of related work offer specific techniques or methods focus on delivering certain EA concerns and offering specific viewpoints for certain interested stakeholders. There is a lack of generic methods that are able to be instantiated in specific techniques or method for modelling different EA concerns/viewpoints. 
- Tooling: The majority of proposals do not provided tools to support an (semi)automatic EA modelling, which make it difficult the demonstration if the proposal really works. This fact leads to the following condition.

- Empirical validation: There is not enough empirical validation in real-life companies to demonstrate the suitability and applicability of the proposed techniques. When such validation exists, it only covers the manual elicitation of EA concerns. Automatic techniques for EA modelling have not been empirically validated.

\section{4 | THE ARCHIREV METHOD}

ArchiRev (whose acronym is built from "enterprise ARCHItecture REVersed" words) is based on a set of reverse engineering techniques aimed at generating ArchiMate models by analysing software artefacts of IS. ArchiRev is defined as a systematization work and can be seen as an extensible method, aligned with the BizDevOps approach., ${ }^{47}$ The main contribution of this research is twofold. First, in a lesser extent, the generic ArchiRev method as a systematization work (see Section 4.1). Second, in a greater extent, the method and developed tool instantiated for items of the three dimensions within ArchiRev (see Section 4.2).

\subsection{The extensible, general ArchiRev method}

In ArchiRev, different software artefacts can be considered as input by using specific and/or adapted reverse engineering techniques which, in turn, can discover and model further EA elements. Different reverse engineering within ArchiRev not only contribute to generate more accurate and complete EA models (i.e., further elements). Additionally, such techniques take into account certain information of IS artefacts to generate specific viewpoints concerning different stakeholders. ${ }^{21}$ It should be noticed that different EA views can be generated from EA models according to the viewpoints. Thereby, information gathered from IS artefacts drives the selection of certain elements to be included in a specific view, as well as some relationships between those elements. In these specific viewpoints, some layout issues could be addressed through reverse engineering techniques included in ArchiRev. Therefore, ArchiRev can be understand as a multidimensional method with three different dimensions that can be considered (see Figure 4). These dimensions are (i) the possible EA viewpoints/concerns that ArchiRev is able to extract

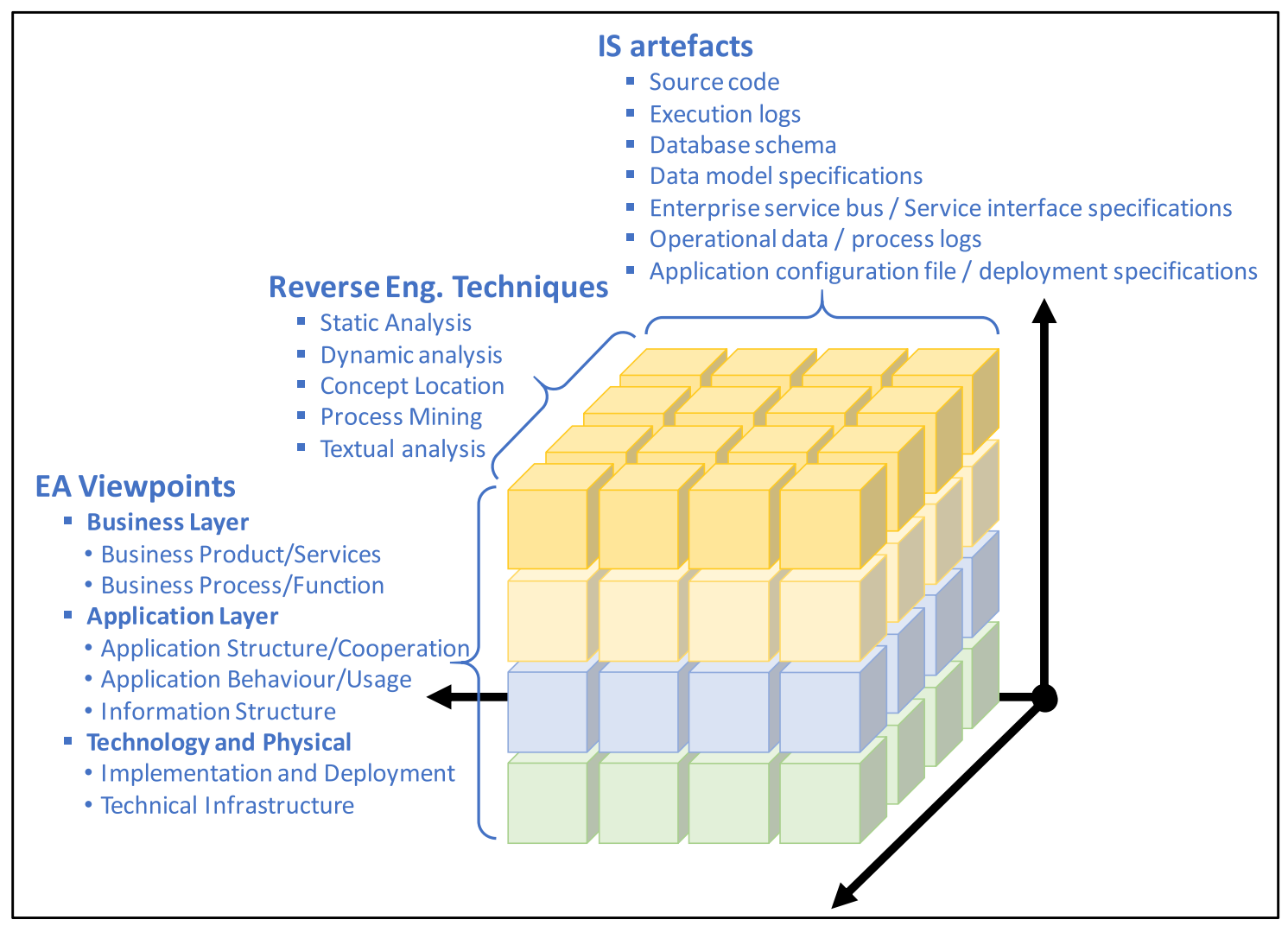

FIGURE 4 Overview of ArchiRev dimensions 
and generate; (ii) the possible reverse engineering techniques that could be used to extract some of those specific EA concerns/viewpoints; and finally, (iii) the dimension of IS artefacts that are considered during reverse engineering for gathering relevant EA information.

According to the ArchiRev cube (see Figure 4), there is no a single reverse engineering technique (or a set of such techniques) to generate the target EA viewpoints. Instead of this, ArchiRev method is thought to instantiate different techniques that, in turn, can consider several artefacts as input for retrieving valuable EA information. The point of this is that all the techniques used in a certain instantiation should be orchestrated to integrate all the relevant EA information that is discovered into a common repository.

This integration not only concerns the combination of gathered information, that is, information that help to complete a certain viewpoint. Actually, information gathered using different reverse engineering technique can be used to discover further, valuable information (e.g., relationships between elements in different viewpoints) that were not able to be discovered using the same reverse engineering techniques in isolation. Let us imagine for example two different reverse engineering techniques used in isolation. On one hand, a source code analyser could generate the application structure viewpoint in the EA. On the other hand, if the deployment specifications for those applications are also analysed, the IT infrastructure could be partially modelled. However, if both techniques are applied in combination (not in a row), relationships like, for example, in which server, a certain application run could be extracted as well.

Despite benefits of combining techniques, not every technique makes sense to be indiscriminately combined with other. Actually, the combination of techniques depends on the considered input IS artefacts as well as the target EA viewpoints to be extracted. According to the classification presented in Lau and Arshad, ${ }^{46}$ possible reverse engineering techniques are static analysis, dynamic analysis, concept location, process mining, or textual analysis, among others.

The second dimension of the ArchiRev cube is the EA viewpoints that can be generated through reverse engineering techniques. A viewpoint in the ArchiMate language is "a selection of a relevant subset of the ArchiMate elements and their relationships. This is the representation of that part of an architecture that is expressed in different diagrams. The most basic type of viewpoint is a simple selection of a relevant subset of the ArchiMate concepts and the representation of that part of an architecture that is expressed in this selection, geared towards the stakeholders that will use the resulting views." ${ }^{14}$

It should be noticed that ArchiRev, as any other reverse engineering methods, has some limitations. There are some EA viewpoints in the strategic layer which are intensive in knowledge provided by human like, for example, goal and principle viewpoint, as well as the organizational structure viewpoint, or motivational viewpoint among other in the most abstracted layers. Although these viewpoints might be accompanied with some information extracted in an automated way, the nature of such viewpoints makes human intervention essential. Despite these limitations, ArchiRev method is able to manage all the remaining EA viewpoints, especially those in the application, technology and physical layers, as well as some viewpoints in the business layer. ${ }^{21}$

The third dimension of the ArchiRev cube is the IS/software artefacts that can be used as input. The usage of each artefact together with certain reverse engineering technique is aimed at generating one (or various) EA views based on previously mentioned viewpoints. Table 1 shows the most common possible combinations between IS artefacts and two previous dimensions.

\subsection{1 | ArchiRev usage scenarios and human support}

ArchiRev is a reverse engineering method that can be used to automate EA models creation. Its automation nature speeds up the modelling activity and prevent experts' subjectivity and error-proneness associated with any manual work. However, as any automated reverse engineering method, ArchiRev has some challenges to be addressed as we mentioned (e.g., semantic loss, impossibility of extract some motivational, and business goals information). As consequence, we previously stablished three different scenarios ${ }^{26}$ in which ArchiRev can be specifically used (see numerated black arrows in Figure 5).

- Scenario 1: IS artefacts are used as input for modelling some EA viewpoints in the application layer by following a bottom-up approach. The lack of semantics in some IS artefacts usually prevent to get EA models at higher abstraction levels such as strategy and business layers. Even so, some EA viewpoints at these levels might be partially modelled when input artefacts enable this.

- Scenario 2: Taking into account limitations of the previous scenario, this alternative scenario follows a top-down approach. The idea is that some existing EA models in the strategy, business (but also motivational) layers can be used in combination with IS artefacts. Some examples of such models are canvas models and business motivation model using BMM (business motivation model) notation. The key of this scenario is the usage of such models to drive EA modelling. One advantage of this scenario is the easy detection of business-IT misalignment issues, which contributes to the whole EAM.

- Scenario 3: Apart from these up-and-down scenarios, horizontal relationships, as proposed by the Enterprise Continuum in TOGAF, ${ }^{47}$ might be considered in later or parallel phases. Thereby, mining techniques as ArchiRev may contribute to EA modelling for a specific organization. In 


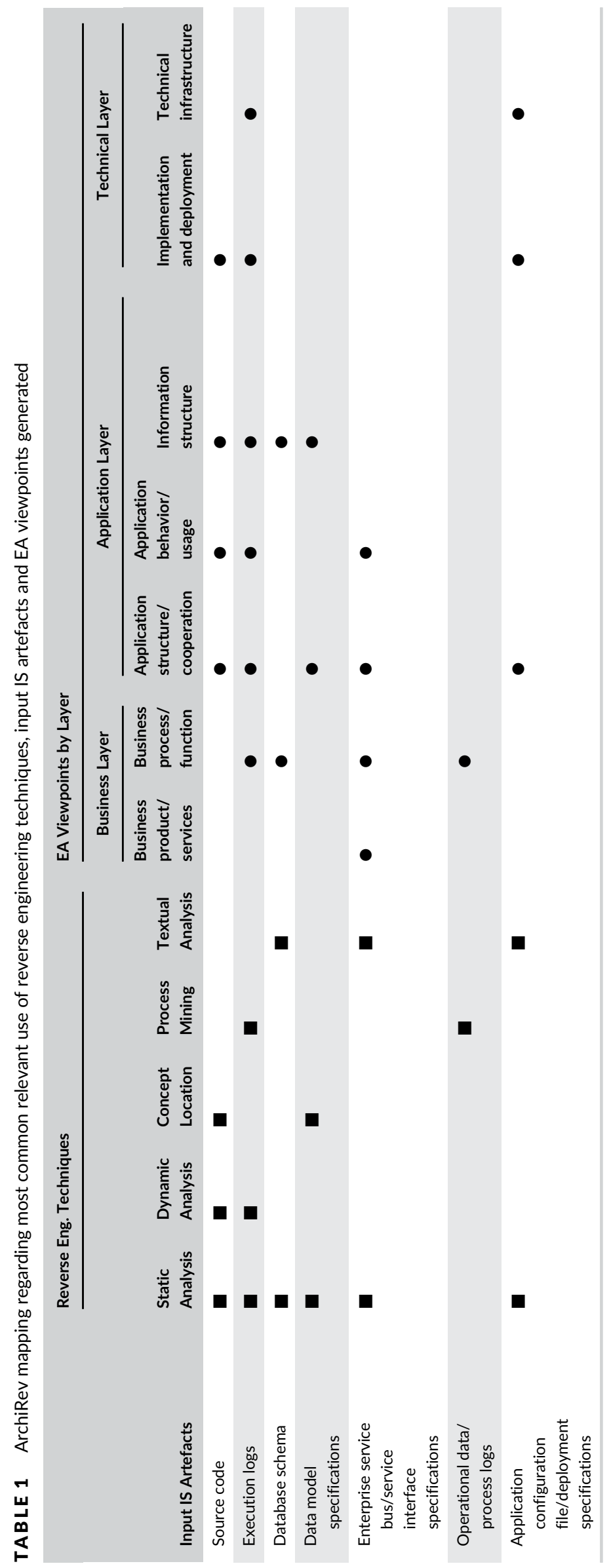


FIGURE 5 Scenarios for applying ArchiRev (adapted from Pérez-Castillo et $\mathrm{al}^{26}$ )

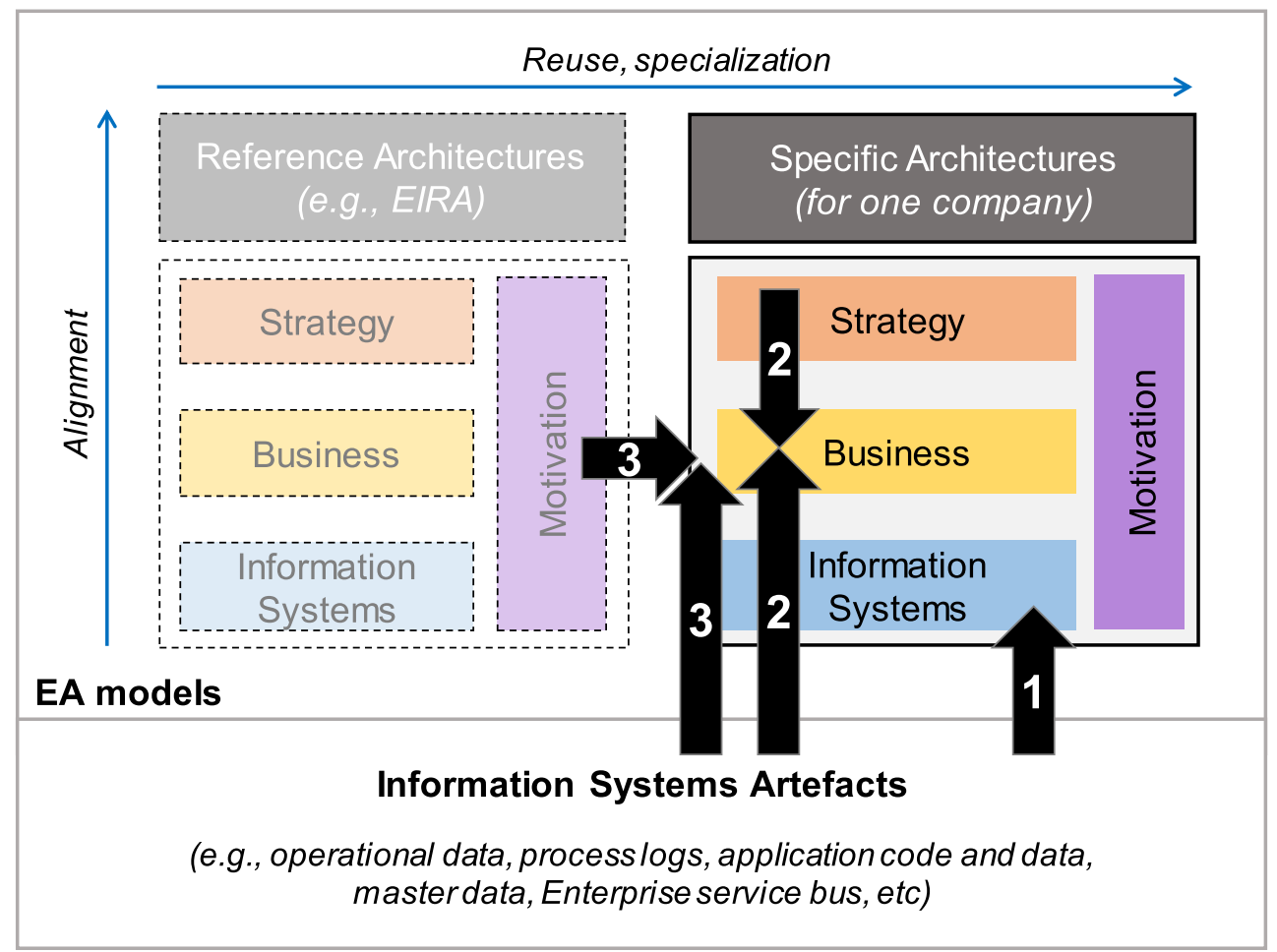

this context, the inputs are the generic EA models of the reference architecture in the strategy, business, and motivation layers, as well as the IS artefacts as used in the preceding scenarios. ${ }^{26}$

In these three scenarios, human intervention is necessary. ${ }^{48}$ Scenario 1 can use it in order to parametrize reverse engineering techniques employed to generate EA models. For example, business and IT experts are responsible for providing the IS artefacts that are used as input.

In scenarios 2 and 3, the human intervention is even more important, because information provided by experts is considered as an additional source of information. These experts can provide information that can be collected by techniques like interviews, focus group, Delphi technique, serious games and, in general, any other technique focusing on experts' information collection. Scenarios 2 and 3 use this information together with other automatic reverse engineering techniques. Thereby, the most critical task is the integration between EA elements discovered automatically and those derived from experts' information.

Apart from the mentioned human intervention, EA architects could be involved in any of the depicted scenarios at the end of the EA modelling process by refining the generated models. In such case, the list of operations an EA architect can do is the following:

- Add missing elements: for example, some EA elements that were not able to be discovered nor modeled.

- Remove irrelevant elements: for example, some false positive elements automatically generated, which do not represent any real EA element in the company.

- Modify elements: for example, rename labels of some elements or change source and/or target element in some relationship elements.

These three operations can be addressed at both the EA model and the EA viewpoint level. However, the EA architect could do other operations exclusively at the EA view level.

- Generate an additional view: A new view could be generated by EA architects to represent some concerns and viewpoint for some specific stakeholders, which have not been automatically generated. In combination with this new viewpoint, some of the previous operations at EA model level could be accomplished first.

- Remove an existing view: A nonrelevant or repetitive view could be discarded, for example, with the goal of reducing the number of views and speed up the EA maintenance process.

- Modify an existing view: This operation is can be achieved by applying the three operations at EA model level previously depicted.

- Split view: An existing view is split in two or more smaller views according to a certain viewpoint.

- Merge views: Two or more existing views are integrated into a single view concerning a specific viewpoint. 


\subsection{ArchiRev instantiation and tool}

This section addresses the specific instantiation of ArchiRev method, by implementing some reverse engineering techniques in a tool. This tool is developed as a web application based on Java which can be accessed online at http://infalarcosj.esi.uclm.es/ArchiRev (see Figure 6).

The architecture of the tool has been designed considering the extensible nature of ArchiRev. As a result, further reverse engineering techniques and IS artefacts could be considered in the future for generating additional EA views. Thus, the process of generating EA models is divided in three steps (see tabs in the screenshot of Figure 6):

- Step 1. Knowledge sources: In the first step, the user has to define the list of knowledge sources to be considered as input during the extraction process. Every source of knowledge is defined by its type (e.g., as defined in the presented, possible input artefacts), and the artefacts itself which are uploaded. Every type of source of knowledge is associated with a certain reverse engineering technique. So far, this tool supports the static analysis of source code, having provided parser for Java and C\# code. Thus, one can select "C\# source code" as artefact type and then upload a zipped file containing all the source code of one application. It should be noticed that several sources of knowledge could be added/uploaded.

- Step 2. Extraction: The second step is about the definition of extraction tasks. Having different sources defined in the previous step, different extraction tasks could be run. This functionality allows users to experiment with the creation of different EA models/viewpoints. For example, in the extraction task 1 , the source of knowledge 1 could be considered with a configuration for generating the EA application structure viewpoint. Additionally, in the extraction task 2, sources of knowledge 1 and 2 could be considered with a different configuration aimed at generating the application structure and information structure viewpoints. In this step, a part of the target EA views to be generated, some configuration can be defined. For example, in case of the static analysers (associated with some source code artifacts), the mapping between some source code elements and some EA elements could be provided.

- Step 3. EA models: After all extraction tasks have been executed, the third step is intended to visualize EA models and all the generated views associated with every model (see Figure 6). Apart from visualizing, in this step, some computed metrics by EA model/viewpoint can be checked and models might be exported using the ArchiMate Model Exchange File Format in order to open and edit the EA model with other EAM suites.

Despite the suite has been designed to be extended according to the ArchiRev method, current version provides a specific default instantiation according to the following parameters regarding the three dimensions of ArchiRev:

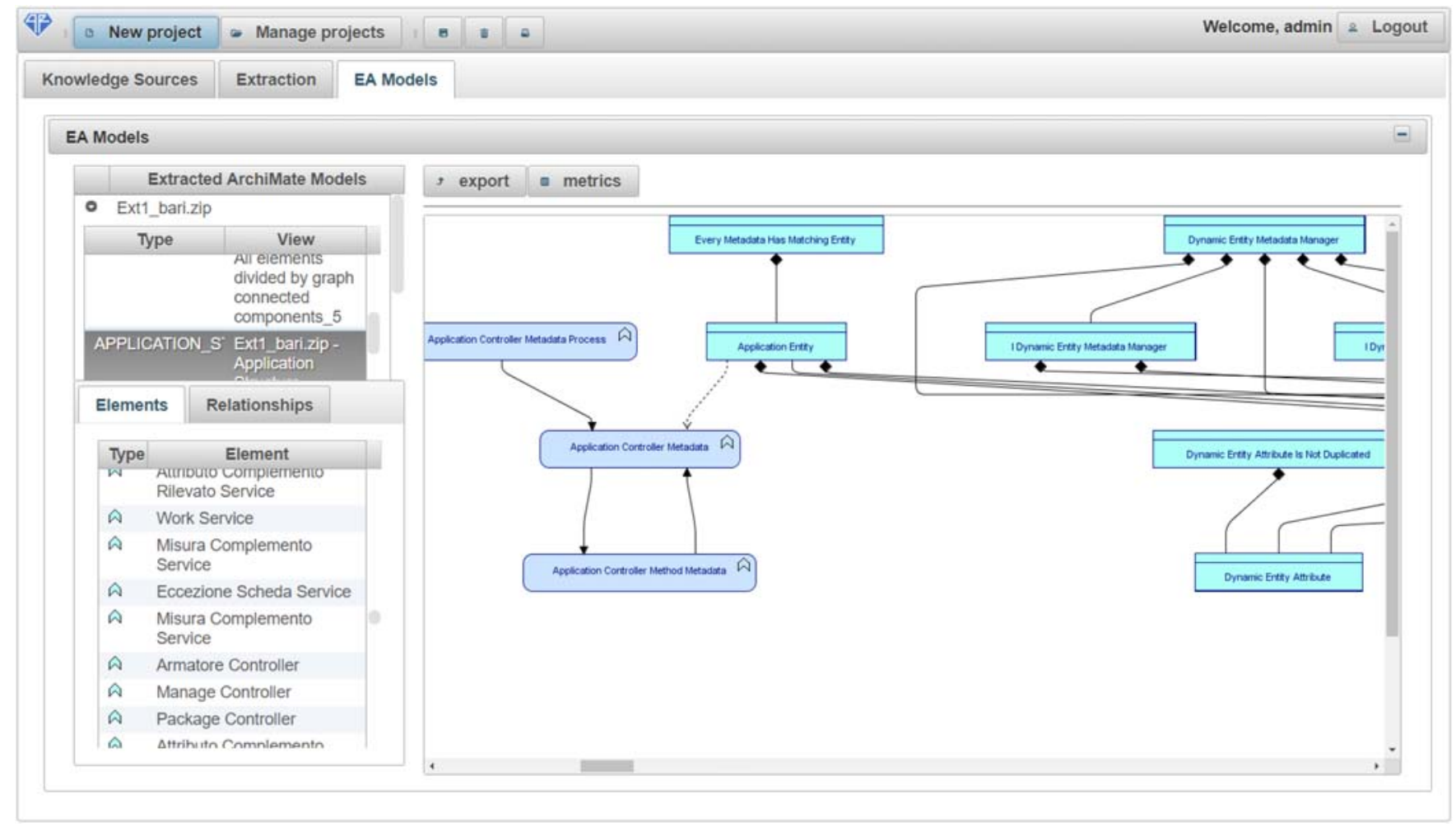


1. Reverse engineering techniques:

- Static analysis

- Textual analysis

- EA viewpoints generated:

- Application structure viewpoint

- Application behaviour viewpoint

- Information structure viewpoint

- Application clusters viewpoint

- IS Artefacts:

- Source code (Java and C\#)

- Data model specifications (as embedded in source code)

As a result, the core logic for generating EA models in an automatic way is supported by the static analyser. This syntactically analyses source code that follows a structured organization and, therefore, can be expressed in terms of a grammar. The parser is in charge of recognizing the whole structure of a piece of source code and generating an abstract syntax tree (AST) from which specific information can be then gather (e.g., callable units belonging to a compilation unit). Then, the tool applies some transformation between some elements in source code and some target ArchiMate elements and relationship between them.

Regarding ArchiMate elements, the parser basically detects all the compilations units and their callable units (respectively, classes and methods in object-oriented programming languages like Java or C\#). Together with these core elements, the parser detects annotations made by every class. Annotations are compilation directives that changes behaviour of classes, which provide a lot of semantics for EA application structure/behaviour views (see Algorithm 1). Together with these annotations, names of classes are additionally analysed by textual analysis in order to recognize some word semantically equivalent to some annotations in case these are not used. This information is used to transform every class (when possible) in a specific ArchiMate element.

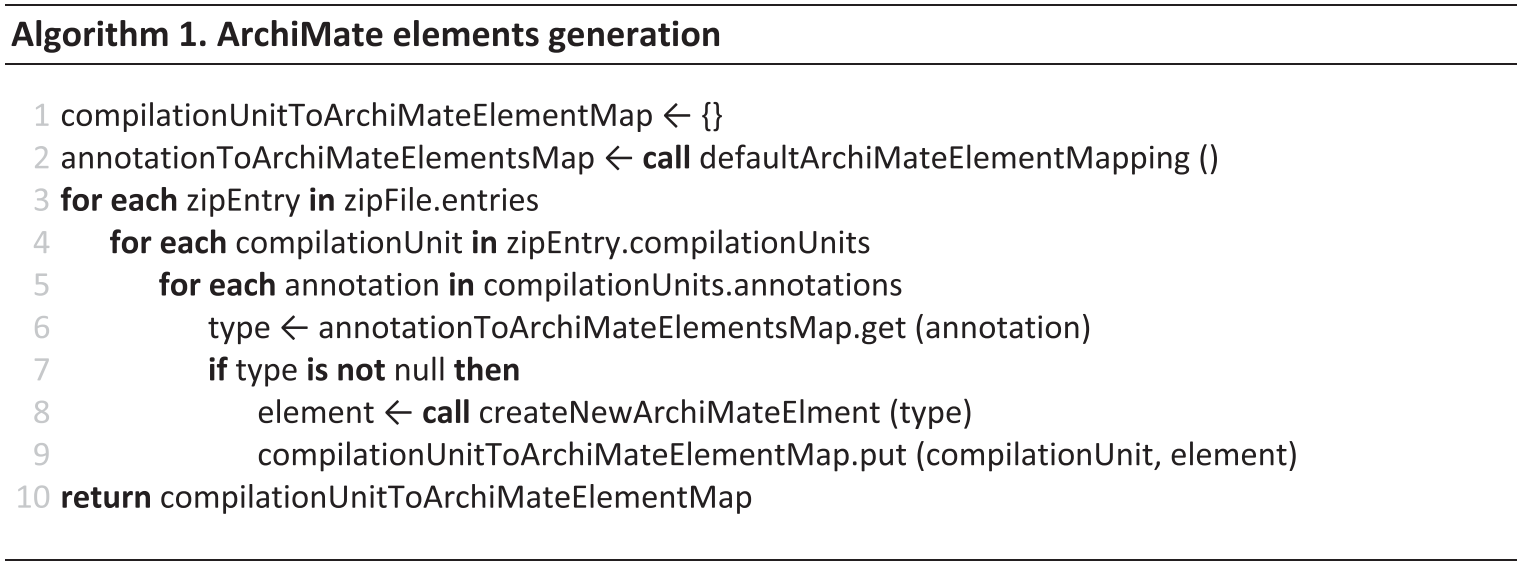

This tool provides a default mapping between these annotations (or words discovered by textual analysis in class names) and ArchiMate elements (see Algorithm 1). However, as we explained before, this mapping could be refined by the user of the tool in step 2 . The default mapping is provided in Table 2 .

Concerning ArchiMate relationships, the parser inspects source code to detect calls between different callable units as well as any usage of object of a certain class. As a result, an ArchiMate relationship is created between the source compilation unit and the target one (both through a call or via object usage) (see Algorithm 2). In this case, several types of ArchiMate relationship could be established although the tool considers the respective ArchiMate elements of both source and target source code elements. Depending of the type of the outgoing ArchiMate elements, the algorithm chooses the most common kind of ArchiMate relationship between such elements (see Table 3). 
TAB LE 2 Default mapping between compilation unit annotations and ArchiMate elements implemented in ArchiRev

\begin{tabular}{|c|c|}
\hline Compilation Unit Annotation & ArchiMate Element \\
\hline ManagedBean & 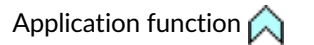 \\
\hline Controller & 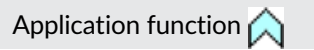 \\
\hline Named & Application function $\widehat{\widehat{W}}$ \\
\hline Service & Application function \\
\hline Table & Data object $\square$ \\
\hline MappedSuperclass & Data object $\square$ \\
\hline Repository & Application component 产 \\
\hline SpringBootApplication & Application component 产 \\
\hline
\end{tabular}

TAB LE 3 Default ArchiMate relationships generated between elements implemented in ArchiRev (source elements in rows and target elements in columns)

\begin{tabular}{lllll} 
& Application Function & Application Component & Application Service \\
\hline Application function & Triggering $\nearrow$ & Triggering & Access & Access \\
Application component & Serving $\nearrow$ & Serving & Triggering \\
Application service & Realization & Realization & Access \\
Data object & Access & Association & Access
\end{tabular}

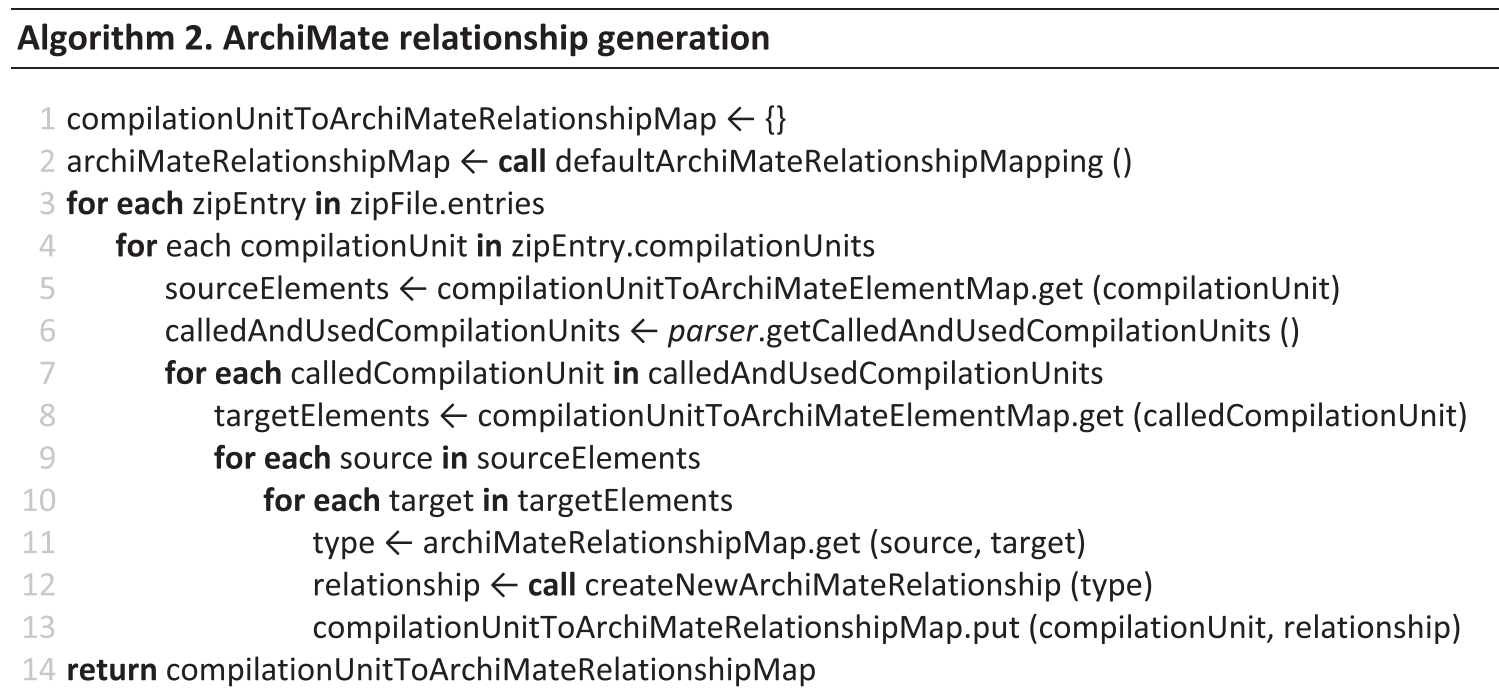

Together with the EA model generation, some views for the obtained model are generated according to the viewpoint selection made by the user. The viewpoint generation consists of two main tasks. First, some elements are filtered in to be included in the view (i.e., a view represents a subset of elements and relationships that will be presented). This filtering is accomplished by simply defining with kind of ArchiMate elements and relationships are able for every viewpoint. Second, all the filtered elements and relationships should be graphically represented in a diagram according to the concrete syntax of ArchiMate. For this second task, the tool uses some open source graphing facilities that allows managing layout and other issues of graph generations.

Mapping between EA elements and pieces of source code, as well as relationships (see Tables 2 and 3 ), has been defined through a conscious study of mentioned related work. However, the tool allows users to configure alternative transformations according to specific domains or technology contexts. 
Apart from the standard views, this tool is able to generate (as a subset of the views presented in Section 4.1); these tools can additionally generate a set of special views that provides smaller diagrams for every atomic connected component of the whole graph represented in the EA model. This set of views is useful when an EA model represents a huge graph with various connected components, which could be useful to have in independent, smaller views.

\section{5 | REAL-LIFE CASE STUDY}

This section presents the industrial case study conducted to empirically demonstrate the feasibility and applicability of the ArchiRev suite developed as an instantiation of the ArchiRev method. The case study has been planned and conducted according to the well-proven protocol proposed by Runeson et al. ${ }^{49}$ This protocol defines at least four stages: background, design, analysis and interpretation and finally validity evaluation.

First, during background stage (cf. Section 3), previous research on the field is identified, which is aimed at defining main research questions that will be addressed during the case study conduction. Second, the design stage (cf. Section 5.1) has to identify the "object of the study" (e.g., a novel method, technique or any other proposal) and the "purpose of the study," that is, the evaluation of some features in which the case study is focused (e.g., the effectiveness, efficiency and scalability). These features are then assessed through the respective measures (typically the dependent variables). The definition of the independent variable is also important and is established in this stage. The independent variable definition has to answer the following interrogations: Is the study a single-case or a multiple-case? Is it an embedded (using several analysis units) or a holistic one? The design stage also explains certain criteria used to select an appropriate case. Finally, it proposes the execution plan to conduct the study as well as the data collection plan for recording all the valuable data (cf. Section 5.1). The third stage (cf. Section 5.2) is devoted to analyse and interpret results. It analyses all the collected data and finds suitable criteria for interpreting main findings achieved. These analyses figure out all the necessary evidence to answer main research questions. Finally, the validity evaluation stage (cf. Section 5.3) attempts to verify that obtained results are unbiased and generalizable for the whole population. In case some threats to the validity are detected, actions for mitigating them are proposed as well.

\section{1 | Case study design}

On the one hand, the object of the case study is the ArchiRev suite (cf. Section 4.2), the artefact designed as a specific instantiation of the ArchiRev method. It should be noticed that the empirical validation of the general, extensible ArchiRev method is outside of the scope of this case study. On the other hand, the evaluation of the effectiveness and efficiency of the developed tool is the purpose of this case study.

The design of this case study is embedded, regarding the Yin's classification. ${ }^{50}$ This is because the study analyses a single case, that is, only one IS. Despite this fact, the IS under study generates several EA views. As a result, the set of such EA views is considered as the analysis unit for this case study.

\subsection{1 | Research questions}

Having defined the object and purpose for the case study, the study evaluates the effectiveness with regard to two different aspects. First, the effectiveness that concerns the mining technique, which can be quantitatively answered in terms of precision, recall and F-measure (see RQ1). Effectiveness is also evaluated regarding the theoretical understandability and modifiability of EA views. The case study also analyses the efficiency of ArchiRev by focusing on scalability of the current version of the ArchiRev tool, that is, through the comparison of the time spent on applying the reverse engineering technique to units with different size (see RQ2). Scalability is important to ensure the tool can work for larger systems and, thus, make its adoption easier.RQ1 Is ArchiRev able to generate EA models with adequate accuracy and completion levels? RQ2 Is ArchiRev able to generate EA models by reverse engineering in a scalable way?

\subsection{2 | Case selection}

This section explains how the IS under study has been selected. This selection is formally carried out through the previous definition of clear selection criteria. The goal is to ensure that the analysis of the selected case is enough to draw strong conclusions afterward. The selection procedure establishes the SC1 to SC3 criteria to choose the most appropriate system: 
- SC1. It must be an enterprise information system. This ensures that the selected system is an IS that performs business operation management. For instance, this criterion discards embedded systems and cross-cutting utility systems, which do not support the business operation of an organization or company.

- SC2. It must be an industrial system. This criterion ensures that the IS is a real-life system that is currently used in the production environment of any company or organization. Thanks to this criterion, all benchmarking and laboratory-made systems are discarded.

- SC3. It must be coded in Java or C\#. This criterion comes from the technical limitation imposed by the current implementation status of the ArchiRev tool.

We should clarify that we did not find a lot of companies interested in providing us with the source code of their business systems. In our opinion, most of the companies we asked did not notice the benefits of conducting the case study, whereas this thought that sharing source code entails many risks. Despite this panorama, we were able to access the source code of some systems developed by the SER\&Practices, an Italian software development company. After analysing available systems according to criteria SC1 to SC3, the case selected was GIST-ERA, an IS of an Italian ship refurbishment company. GIST-ERA allows to manage and plan all the exact measurements that must be taken for every cabin. The particularity of huge cruise ships is that the size of cabin changes over time because of the metallic structure and continuous dilatation and contraction of this. Hence, when a ship is going to be refurbished, all cabins have to be measured completely in order to exact pieces and accessories for every cabin. Together with this, there are other logistic constraints. For example, measurements have to be taken when no customers are inside, or ship is docked in the harbour. These facts make the planning this measurement so critical (see Figure 7).

GIST-ERA follows a server-client architecture. A client application for tablets allows the staff to register all the measures as well as help them to follow an optimal process preventing mistakes. The server side collects all the measures and manages them optimizing material and accessories orders. Technologically, the system is written in C\# and uses an MS SQL Server as storage system. Additionally, this system was coded by using Dynamic MVC framework, which helps to produce data-driven Model/View/Controller applications. This framework also provides some ORM (object-relation mapping) facilities. Unfortunately, due to the fact that researchers had to sign a nondisclosure agreement, the source code cannot be disseminated. However, Table 4 provides some relevant object-oriented code metrics.

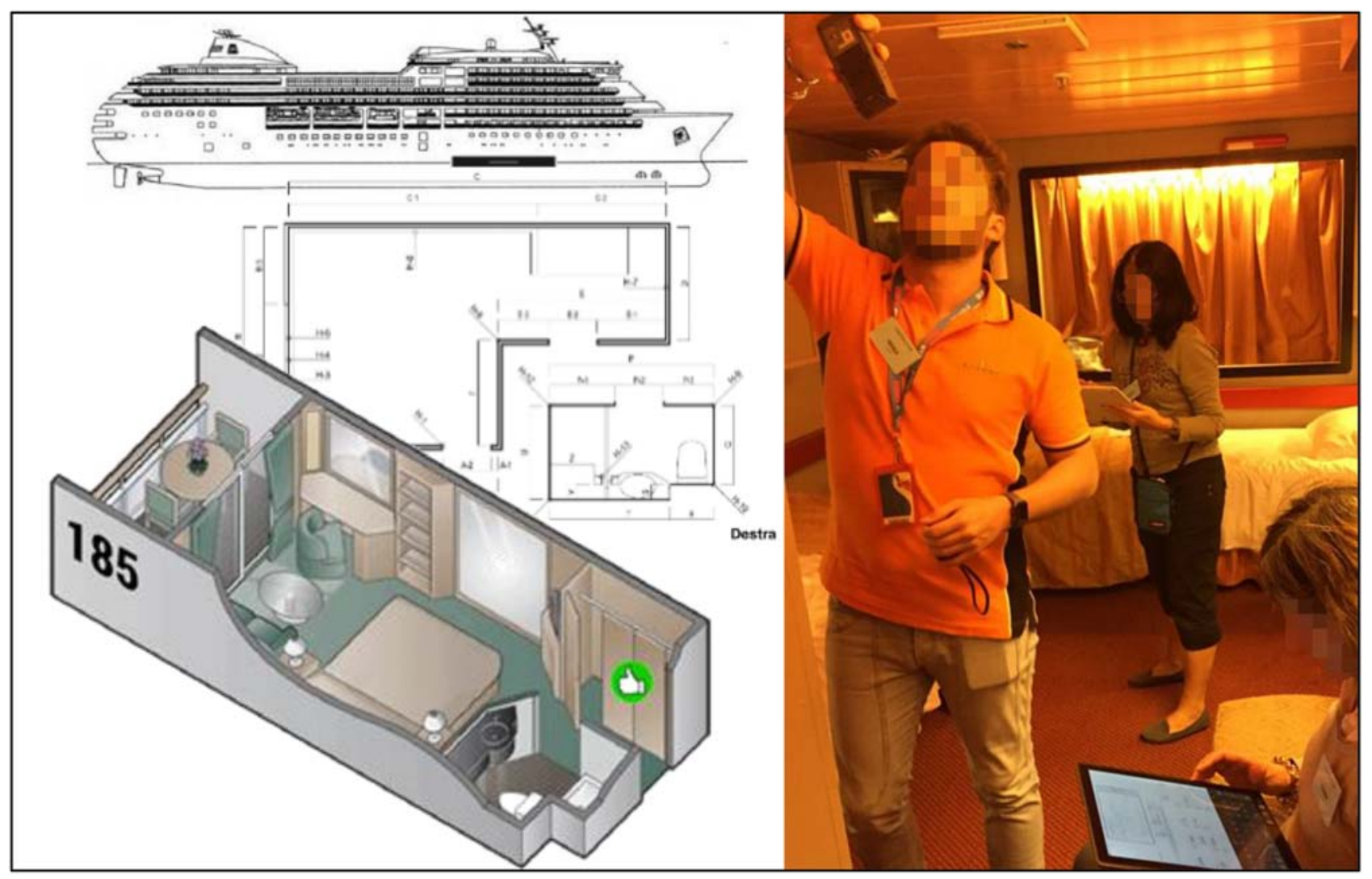

FIGURE 7 Representation of the business core supported through the IS of the ship refurbishment company 
TAB LE 4 Some relevant object-oriented metrics for the system under study

\begin{tabular}{|lccc|}
\hline Metric & Total Number & Average & Standard Deviation \\
\hline \# LoC & 37831 & & - \\
\hline \# Classes & 414 & - & - \\
\hline \# Nonabstract methods on classes & 2243 & 6.35 & - \\
\hline Public methods on classes & & 1.91 & 9.29 \\
\hline Cyclomatic complexity on methods & - & 10.17 & 3.16 \\
\hline Afferent coupling on assemblies & - & 63.25 & 17.51 \\
\hline Efferent coupling on assemblies & - & 1.70 & 31.15 \\
\hline Relational cohesion on assemblies & - & 0.85 & 0.76 \\
\hline Instability on assemblies & - & 0.21 & 0.22 \\
\hline Abstractness on assemblies & & 0.15 \\
\hline
\end{tabular}

\subsection{3 | Variables}

Independent and dependent variables for answering research questions must be defined. The independent variable of this study is the id (a nominal variable) of every EA view generated, which is the analysis unit according to the case study design (see Table 5).

In order to answer RQ1, precision and recall measures are used to evaluate the effectiveness of the ArchiRev method. Both measures have been used in retrieval information scenarios as well as for assessing business process archaeology methods. ${ }^{51}$ The precision measure indicates the amount of relevant recovered elements within the set of recovered elements in a business process model. An element is considered relevant if it faithfully represents a business element in the real world. The recall measure represents the fraction of relevant recovered elements of the total of relevant (recovered and not recovered) elements. Because there is an inverse relationship between the precision and recall measures, these measures are usually not evaluated in an isolated manner. Instead of this, these measures are combined into a single measure known as F-measure, which consists of a weighted harmonic mean for previous measures. These two measures are considered as dependent variables of the case study concerning RQ1 (see Table 5). Precision and recall are usually computed by assessing the retrieved model against an oracle (i.e., the expected model). In EA there is extremely difficult to find a company with EA models (specially with updated ones). Hence, even if there is not an oracle, precision and recall can be computed through the assessment by experts. This means that the oracle is built during the evaluation, through the identification of true/false positives and false negatives. Thus, workshops with experts from the organization where conducted to assess the EA models delivered. Such experts determined missing and wrong elements (both nodes and edges) in order to compute precision and recall.

TAB LE 5 Variables definition

\begin{tabular}{|c|c|c|c|c|}
\hline Name & Definition & Type & $\begin{array}{l}\text { Scale } \\
\text { Type }\end{array}$ & Range \\
\hline EA view & Each EA view generated & Independent & Nominal & \\
\hline Recall & $\frac{\mid\{\text { relevant elements }\} \cap\{\text { recovered elements }\} \mid}{\mid\{\text { relevant elements }\} \mid}$ & Dependent & Interval & $x \in[0,1]$ \\
\hline F-measure & $\frac{2}{\frac{1}{\text { Precision }+\frac{1}{\text { Recall }}}}$ & Dependent & Interval & $x \in[0,1]$ \\
\hline Density & $\frac{|E|}{\frac{|V|(|V|-1)}{2}}$ & Dependent & Interval & $x \in[0,1]$ \\
\hline Parsing time & Time spent on applying reverse engineering to source code & Dependent & Scale & $x \in \mathbb{R}^{+}$ \\
\hline $\begin{array}{l}\text { Model generation } \\
\text { time }\end{array}$ & Time spent on generating EA model items & Dependent & Scale & $x \in \mathbb{R}^{+}$ \\
\hline $\begin{array}{l}\text { Compilation unit } \\
\text { size }\end{array}$ & $\begin{array}{l}\text { The size in lines of source code of each compilation unit of the source code under } \\
\text { analysis. }\end{array}$ & Independent & Scale & $x \in \mathbb{R}^{+}$ \\
\hline
\end{tabular}


Together with those variables, in order to achieve a better assessment of the effectiveness in RQ1, some measures are additionally considered. These are three well-proven measures proposed by other works ${ }^{52,53}$ : size, connectivity, and density, which are theoretically related with the understandability and modifiability of models. Because the generated EA models have to be manually tuned by experts, it is important to get understandable and modifiable models. Additionally, the study of the correlation of these variables against precision and recall might help to improve the design and development of similar techniques.

In order to define these measures in a formal way, we can consider a business $E A$ view represented as a graph through the notation $G=(V$, $E$ ), being $v_{1}, v_{2} \in V$ (vertices or nodes) and e $\epsilon E$ (edges or arcs between nodes), $e=\left(v_{1}, v_{2}\right)$ (see Table 5). Size is the number of nodes in an $E A$ view (i.e., those defined in Table 2). The higher the size is, the poorer understandability perception is for a certain EA view. Connectivity is computed as the proportion of total number of edges in an EA view (i.e., those defined in Table 3) regarding the total number of nodes. Both understandability and modifiability are negatively impacted by higher connectivity ratios, that is, lower connectivity values corresponds to EA views that are not highly intricated and, therefore, can be understood and maintained in an easy way. ${ }^{54}$ Lastly, the ratio between the total number of edges in an EA view and the maximum number of possible edges (theoretically calculated) is known as density. EA views with higher density values are also less understandable and modifiable. ${ }^{54}$

In order to evaluate RQ2, four time-related measures are considered. First, the time spent on analysing every compilation unit of the IS artefact during the application of the reverse engineering technique. Second, the time spent on generating every item inside an EA model. These two times, in some analyses, are differenced for both ArchiMate elements and relationships, because these two kinds of model items are defined in two different steps (see Algorithms 1 and 2). Third, the time spent on generating every view as a subset of model elements and relationships of the EA model. Finally, total time spent on generating every EA view is considered as the sum of the three previous measures.

\subsection{4 | Execution procedure and data collection}

The execution procedure consisted of the following steps. All the data collected during the conduction of this case study is online available at Pérez-Castillo. ${ }^{55}$

1. EA models and EA views are mined from the source code of the system through ArchiRev tool. Thus, seven (7) different EA views are collected from the information system under study, which represents the independent variable (see Table 6).

2. The tool automatically computes size, connectivity and density for each view. Such information was moved to a common dataset for the case study.

3. EA models and views are exported as images in order to evaluate precision, recall and F-measure. The base measures for computing those have to be assessed based on experts' judgement (because there is not an existing EA model in the organization). For this duty, we consider two experts (The chief information officer [CIO], with a more technical profile, and the chief operation officer [COO], with a higher business vision). In order to address the disagreements, we use the Delphi technique, ${ }^{56}$ which allows achieving a workable consensus within time limits. We established a workshop with both experts to discuss the generated EA models and, in some cases, they decided to add missing elements. All the existing ArchiMate elements and relationships were analysed one by one, and some of them were discarded. Based on the ArchiMate elements that are added and removed, precision, recall and F-measure are then computed. This data collection is made for elements as well as for relationships in the analysed model/view. In this way, disaggregated values are considered during the discussion phase.

TAB LE 6 Some relevant object-oriented metrics for the system under study

\begin{tabular}{|c|c|c|}
\hline Id & Name & Description \\
\hline 1 & ALL & This view represents the whole EA model, that is, all elements and relationships are included. \\
\hline 2 & APPLICATION_STRUCTURE & $\begin{array}{l}\text { It represents how the information systems is structured. Hence, this view only includes application } \\
\text { functions, application components and all possible relationships between them. }\end{array}$ \\
\hline 3 & INFORMATION_STRUCTURE & $\begin{array}{l}\text { It represents the data and information passive objects. This means, data objects, associations, } \\
\text { aggregations and compositions are the only elements in this view. }\end{array}$ \\
\hline 6 & ALL_CONNECTED_COMPONENT_5 & \multirow{2}{*}{$\begin{array}{l}\text { This set of views are obtained by applying the detection of connected graph components in the whole } \\
\text { model we mentioned previously. The algorithm generates four different views for clusters } 1,3,5 \text { and } \\
7 \text { whereas other clusters were ignored because of small number of elements. }\end{array}$} \\
\hline 7 & ALL_CONNECTED_COMPONENT_7 & \\
\hline
\end{tabular}


4. The tool was modified to be able to register the time spent in applying the static analysis to every individual compilation unit. The time for EA view generation is provided by the tool. This measurement regarding RQ2 is also collected in the common dataset.

5. Having finished the execution, the analysis procedure is conducted by using all the collected data to extract evidences and answer the main research questions (see Section 5.1.5).

6. To conclude, main insights extracted from the study are discussed whereas possible threats to the validity are assessed (see Sections 5.2 and 5.3).

\subsection{5 | Analysis procedure}

The analysis procedure consists of two different types of analyses in order to draw insights concerning research questions formulated previously. The following list summarizes the analysis methods used.

- Descriptive statistics is first used to describe and analyse data by using some numerical and graphical methods.

- RQ1. The expected result is that measured values (precision, recall, F-measure, size, connectivity, density, time, etc.) after the application of ArchiRev are in the appropriate intervals.

- RQ2. It is preliminarily used to check the scalability. Thus, relationship between size and generation time variables is examined through a scatterplot.

- Regression model: Relationships among variables are computed through this analysis. The linear regression model considers the two measures previously depicted as the dependent variable and the independent one. The correlation coefficient $\rho$ (which is between -1 and 1 ) is computed by applying Pearson's rank correlation test. Pearson's rho $(\rho)$ is the degree to which the actual values of the dependent variable are near to the predicted values.

- RQ1. This method is used in RQ1 to check correlations between structural measures (i.e., size, connectivity and density) and retrieval measures (i.e., precision, recall and F-measure).

- RQ2. This linear regression model considers the two time-related measures previously depicted as the dependent variable, and the size as the independent one (both compilation unit size and EA view size, respectively).

\subsection{Analysis and discussion}

This section presents the analysis of the results obtained after the conduction of the case study. This analysis is divided into the two research questions.

\subsection{1 | RQ1: Effectiveness}

Figure 8 shows some descriptive statistics generated with the number of elements obtained. By analyzing these results, we can figure out that the elements of the model generated are application functions (65\%) and data objects (35\%). Then, most of the application function elements were connected among them by triggering relationships (which, in somehow, represents the sequence flow of such functions). Also, application function elements (according to ArchiMate) were connected with data objects by means of access relationships. Finally, the only relationships among data objects is the composition. These results can be directly checked by observing the generated EA views. ${ }^{55}$

Table 7 provides all the measures collected for the ArchiMate views generated. It should be noticed that the id (first column) match with the id of every view depicted previously in Table 6. EA views were generated with a size varying between 6 (the smaller graph component, i.e., view 4) and 132, the maximum number of elements in the model (view 1 with all the elements and relationships). Regarding connectivity, results show that connectivity is between 1 and 2, this means that every node is associated with 1 or 2 relationships on average (this is also shown in top-left plot in Figure 8). Actually, density is low on average (0.20) that represents the amount of relationships generated regarding the theorical total of possible relationships. On one hand, it is appropriate to keep intricacy in low levels and as a result understand and maintain the view in an easier way. On the other hand, after experts evaluated the model, they added up to 47 relationships. Hence, it could be though that the extraction method needs to be tuned to extract more relevant relationships by inspecting the source code or any other IS artefact. Distribution of connectivity and density can be checked in the box plot presented in left side of Figure 9.

Table 7 also shows the results for precision, recall and F-measure, graphically represented in Figure 9 (right side). Distribution of such measures, as well as the mean and standard deviation presented in Table 7, is not the most representative. These values are computed through the 


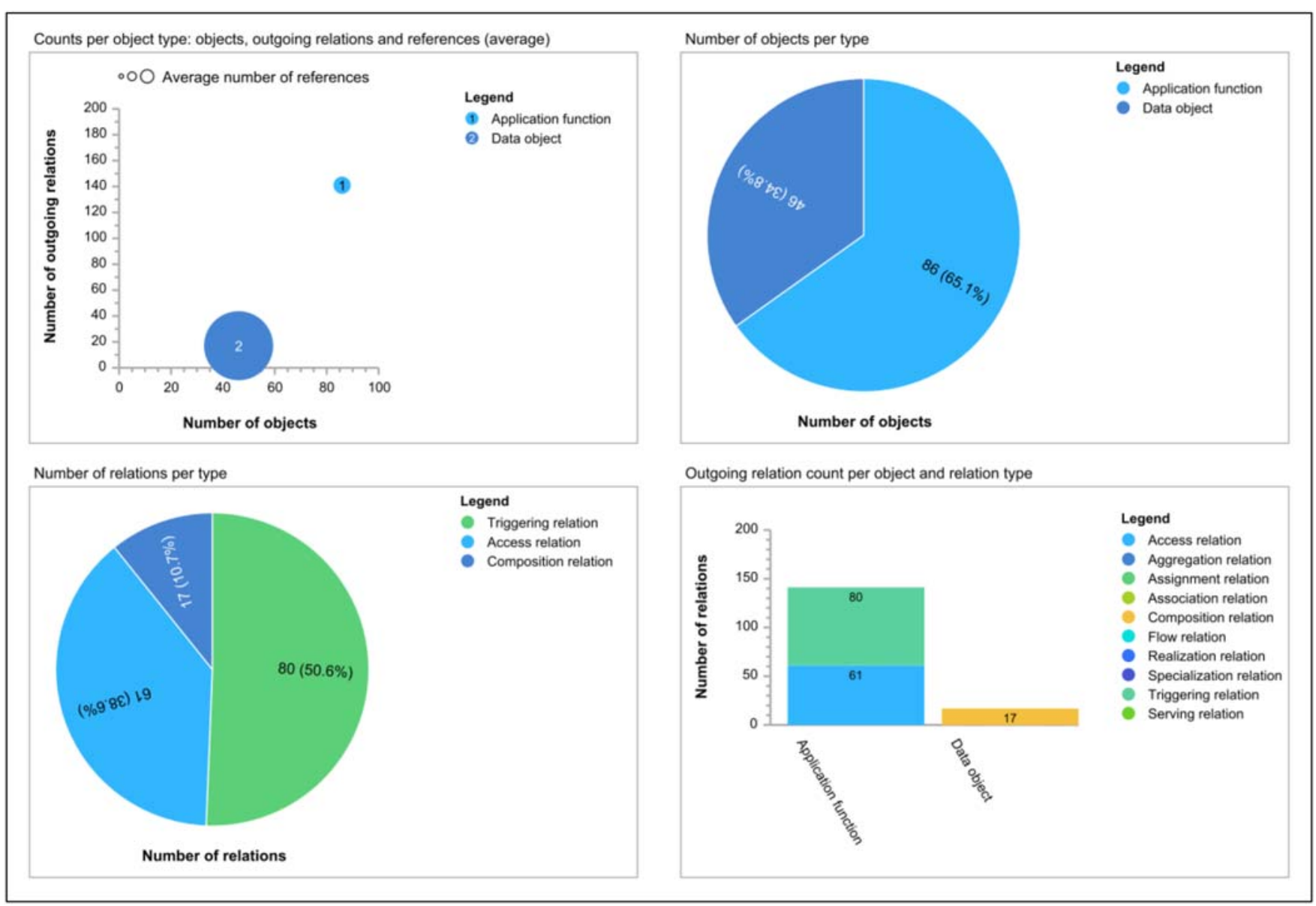

FIGURE 8 ArchiMate model analysis dashboard

views obtained. This means, in case of having other views, different results could be obtained. For this reason, the most important values are those obtained for the view 1 because this view contains all the elements and relationships of the underlying EA model. In such view, the total measures were precision $68 \%$, recall $80 \%$ and F-measure $73 \%$. These values are high which demonstrated that the method is able to discover and generate ArchiMate models. In some of the viewpoints generated from the isolated clusters, the values were different (rows 4 to 7 in Table 7 ). Actually, some of them reported very low levels of precision and recall. This is due to the fact that these isolated clusters were smaller, and most of the elements were discarded by the experts.

In general terms, it should be noticed that precision is slightly lower than recall. This means, that ArchiRev is able to extract most of the relevant elements although some of these elements are non-relevant. The derived insight is that the effort during the manual intervention phase should be more focused on discarding wrong elements rather than adding missing ones. This is what actually happened were the experts analysed the ArchiMate views.

In case these results are disaggregated by elements and relationships, precision and recall follow the same trend (see Table 7), that is, recall is slightly better than precision. However, values obtained for elements were much better than those for relationships (precision: 65\% against $38 \%$, recall: $99 \%$ against 73\%, and F-measure: $72 \%$ against $42 \%$ ). These results mean that ArchiRev works better for element discovering from source code in comparison with relationships. It can be explained because there are some relationships between application functions and data objects that are not represented in source code, or when such relationships are in source code, these are blurred (e.g., through derived associations that are dynamically instantiated during code execution).

Regarding the retrieved "false positive" elements, most of them were elements generated from the inspection of technical/auxiliary source code or even self-generated code derived as a result of the usage of certain frameworks during development. It is important to note the impact of all of these frameworks because many "false positives" could be avoided through the addition of some exclusions tags that can be defined during the extraction parametrization in the tool. So it strongly advisable to include technical experts who knows the IS and eventually discuss this kind of aspects.

Anyway, insights obtained after analysing precision and recall, which were derived after experts' opinion, are in line with connectivity and density values, that is, few relationships regarding the number of elements (see left plot in Figure 9). Actually, a Pearson correlation test was 


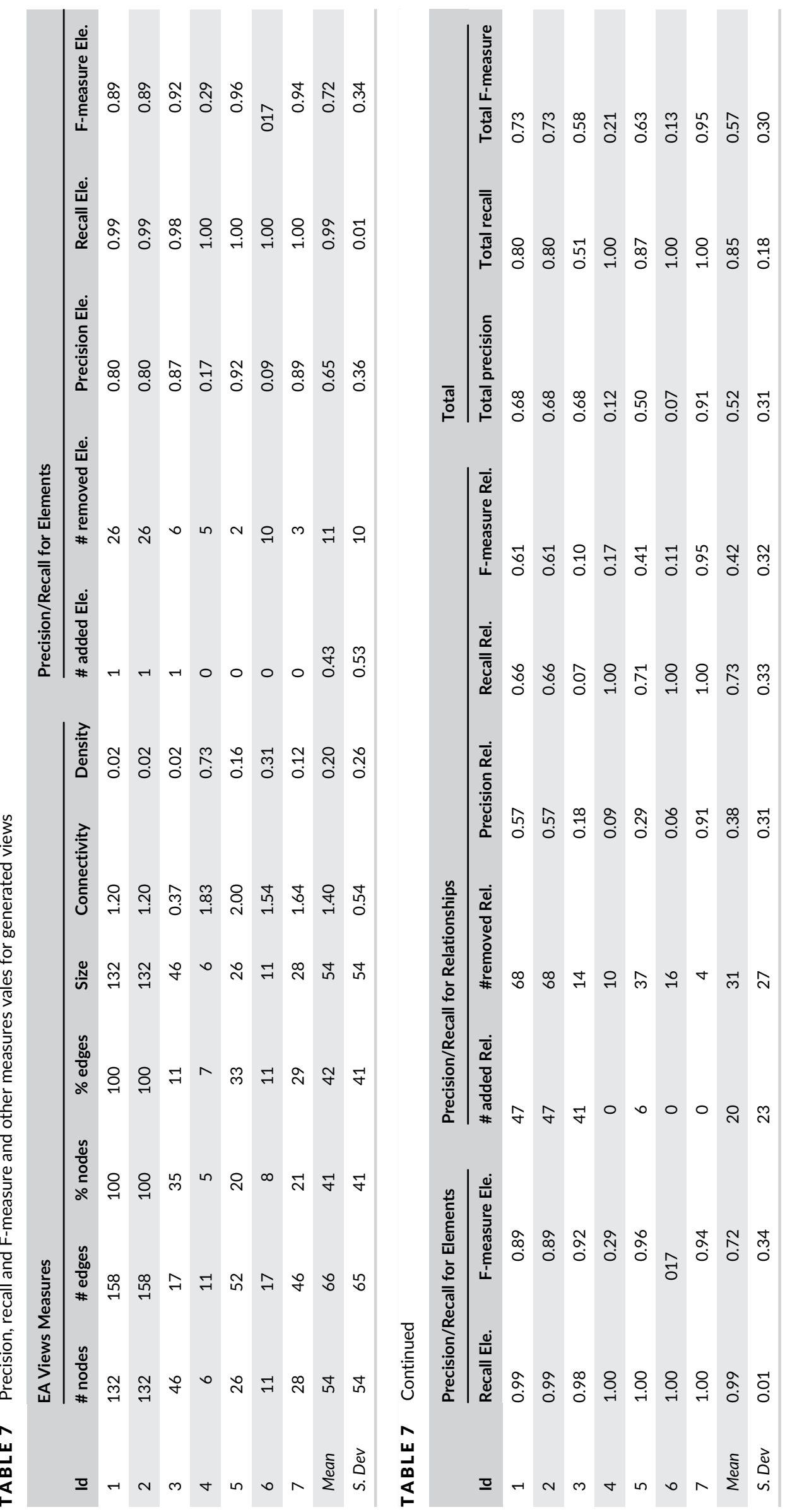




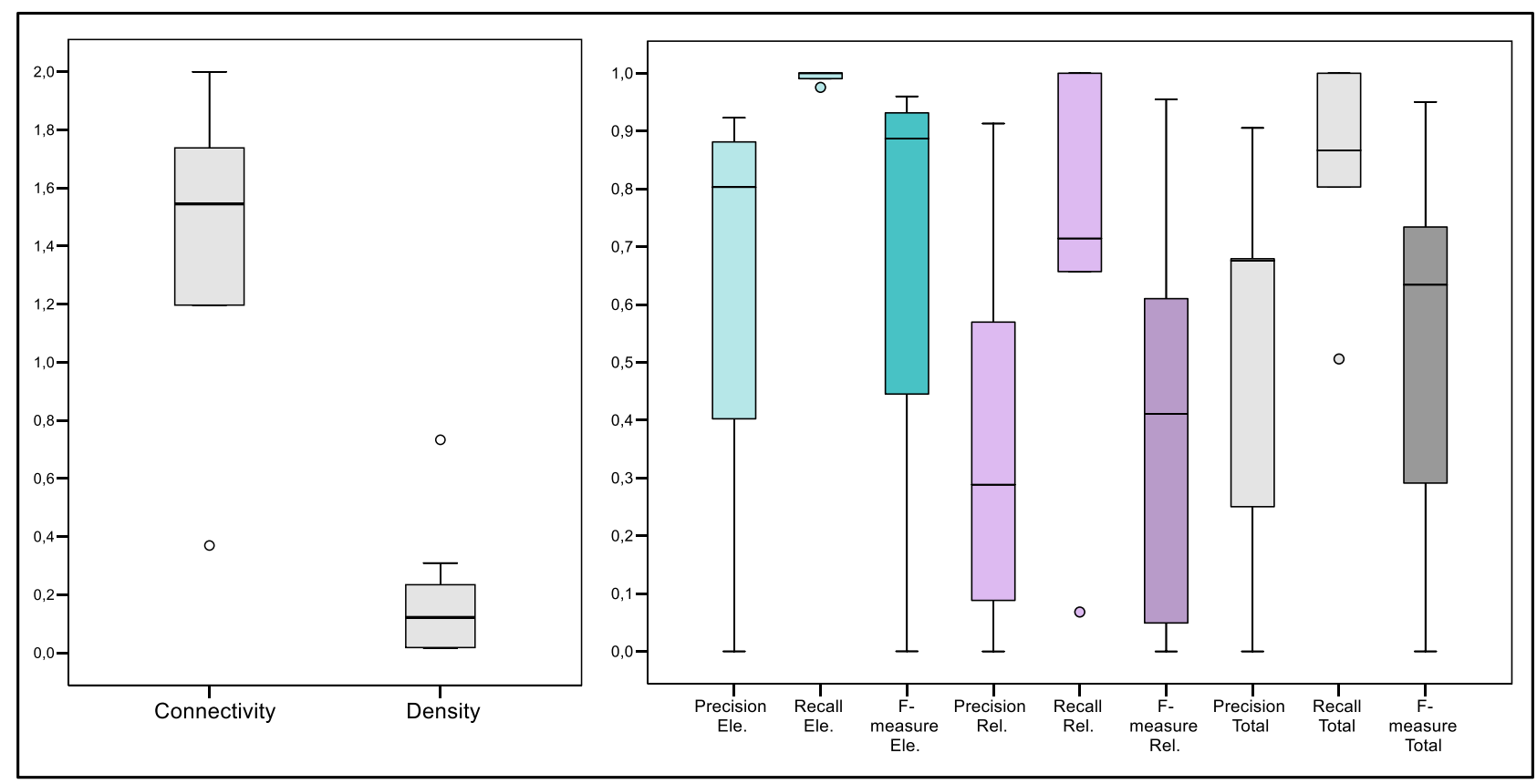

FIGURE 9 Distribution of the measures for the ArchiMate views generated. Connectivity and density (left side) and precision, recall and Fmeasure (right side)

performed for size, connectivity and density with regard to precision, recall and F-measure (see Table 8). The results show that connectivity was related to recall in all the cases (elements, relationships and combination of both). Density, however, presented an inverse linear relationship regarding precision and F-measure, although in this case, there were statistic significant differences for measures taken only for relationships. Of course, size is not correlated with none of these measures as it is expected. These quantitative analyses confirm the mentioned insights based on qualitative analysis. Although size, connectivity and density are more associated with the retrieved model rather than the retrieving method, the study of those measures is important in order to improve ArchiRev by taking into account their influence.

In conclusion, precision, recall and F-measure were around 75\% for the whole ArchiMate model (see first row in Table 7). With these data, up to a certain extent, RQ1 can be positively answered, that is, ArchiRev is able to extract ArchiMate model by reverse engineering. However, as the result shows, human intervention is still necessary to improve the EA models by adding and removing some elements and relationships.

\subsection{2 | RQ2: Efficiency}

This section analyses the efficiency of the ArchiRev method and, in particular, its specific instantiation through the tool presented before. Figure 10 shows two box plots representing the distribution of times. Figure 10 (left side) shows the parsing and generation times for elements (vertices) and relationships (edges). This plot shows that the time spent of parsing source code files regarding generation of elements into the EA model took almost the $99 \%$ of the EA model generation time. The first insight is, therefore, that the time of storing ArchiMate elements information into an EA model file is neglectable. Focusing on parsing time, Figure 10 (left side) also shows that there is no almost difference between the time spent on parsing code for extracting elements information or for discovering ArchiMate relationships. Both measures seem to

TAB LE 8 Pearson correlation test results of structural measures with regards to precision, recall and F-measure

\begin{tabular}{|c|c|c|c|c|c|c|c|c|c|c|}
\hline & & $\begin{array}{l}\text { Precision } \\
\text { Ele. }\end{array}$ & $\begin{array}{l}\text { Recall } \\
\text { Ele. }\end{array}$ & $\begin{array}{l}\text { F-measure } \\
\text { Ele. }\end{array}$ & $\begin{array}{l}\text { Precision } \\
\text { Rel. }\end{array}$ & $\begin{array}{l}\text { Recall } \\
\text { Rel. }\end{array}$ & $\begin{array}{l}\text { F-measure } \\
\text { Rel. }\end{array}$ & $\begin{array}{l}\text { Total } \\
\text { precision }\end{array}$ & $\begin{array}{l}\text { Total } \\
\text { recall }\end{array}$ & $\begin{array}{l}\text { Total } \\
\text { F-measure }\end{array}$ \\
\hline Size & $\rho$ & 0.489 & -0.415 & 0.528 & 0.491 & -0.341 & 0.475 & 0.524 & -0.387 & 0.540 \\
\hline \multirow[t]{2}{*}{ Connectivity } & $\rho$ & -0.322 & 0.964 & -0.336 & -0.012 & 0.850 & 0.113 & -0.388 & 0.873 & -0.228 \\
\hline & Sig. & 0.481 & 0.000 & 0.461 & 0.980 & 0.016 & 0.809 & 0.389 & 0.010 & 0.623 \\
\hline
\end{tabular}



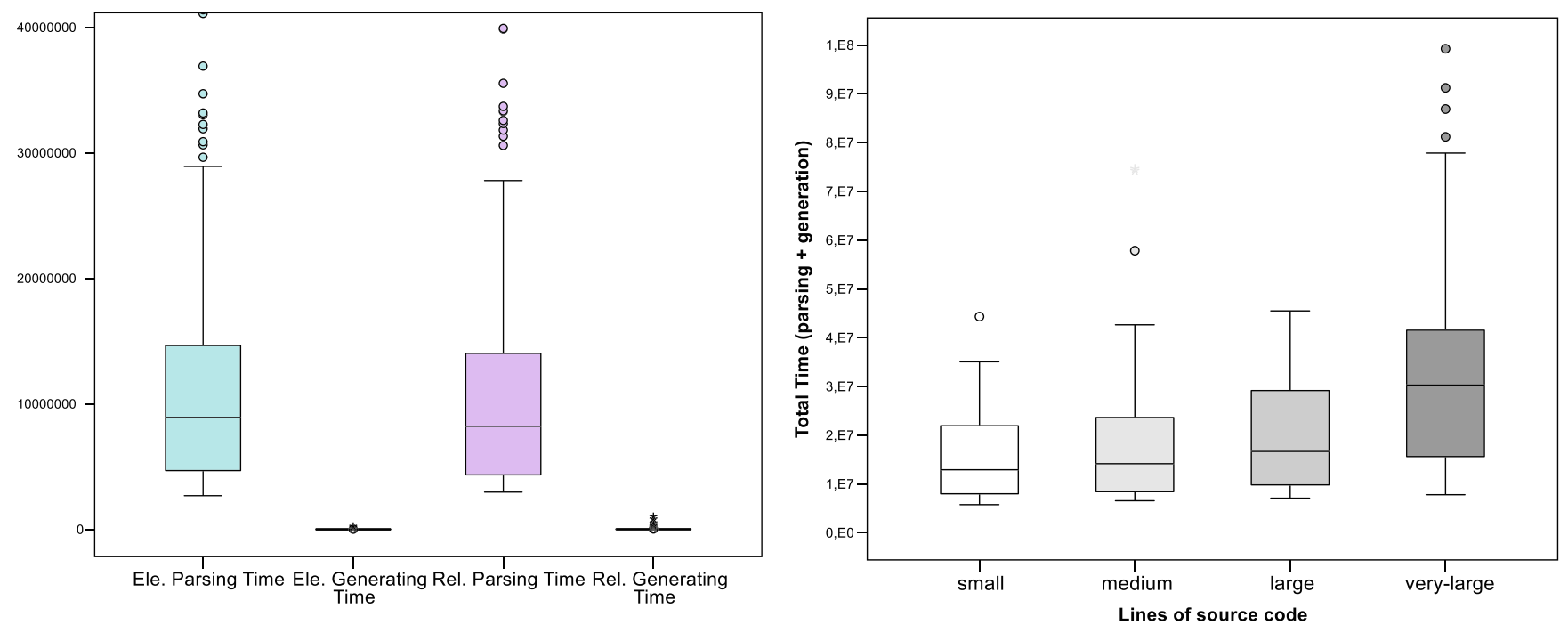

FIGURE 10 Box plots for time analysis. Left, parsing and EA model elements generation times divided by ArchiMate elements and relationships. Right, total time for EA model generation categorized by size of the compilation units

follow the same distribution. Figure 10 (right side) shows the total time for ArchiMate model generation by considering size of the compilation unit under analysis. This plot considers values small, medium, large, and very large according to the quartiles of the distribution of lines of source code for every compilation unit. This pot shows that there are some differences in time regarding the size, specifically when size is very large.

The question to be answer in this point is if this increase is linear regarding the number of lines of source code. In case the answer was negative, we may not say the method is scalable. As a result, Table 9 shows a matrix with the correlation values (according to the Pearson test) between the time measures and the independent measures that can affect those. Cells with values close to 1 and statistically significant are highlighted. As such values show, there is a positive linear correlation regarding lines of source code (LoC) with parsing time and total time, with values around $80 \%$. These results are graphically stated through the scatter plots depicted in Figures 11 and 12).

Although a linear correlation exists for parsing time measures, EA model generation time is not correlated: However, it was shown (see Figure 10) that this time is neglectable. Hence, the randomness effect probably affected results of this test with regard to that measure. In fact,

TABLE 9 Pearson correlation test results for efficiency measures

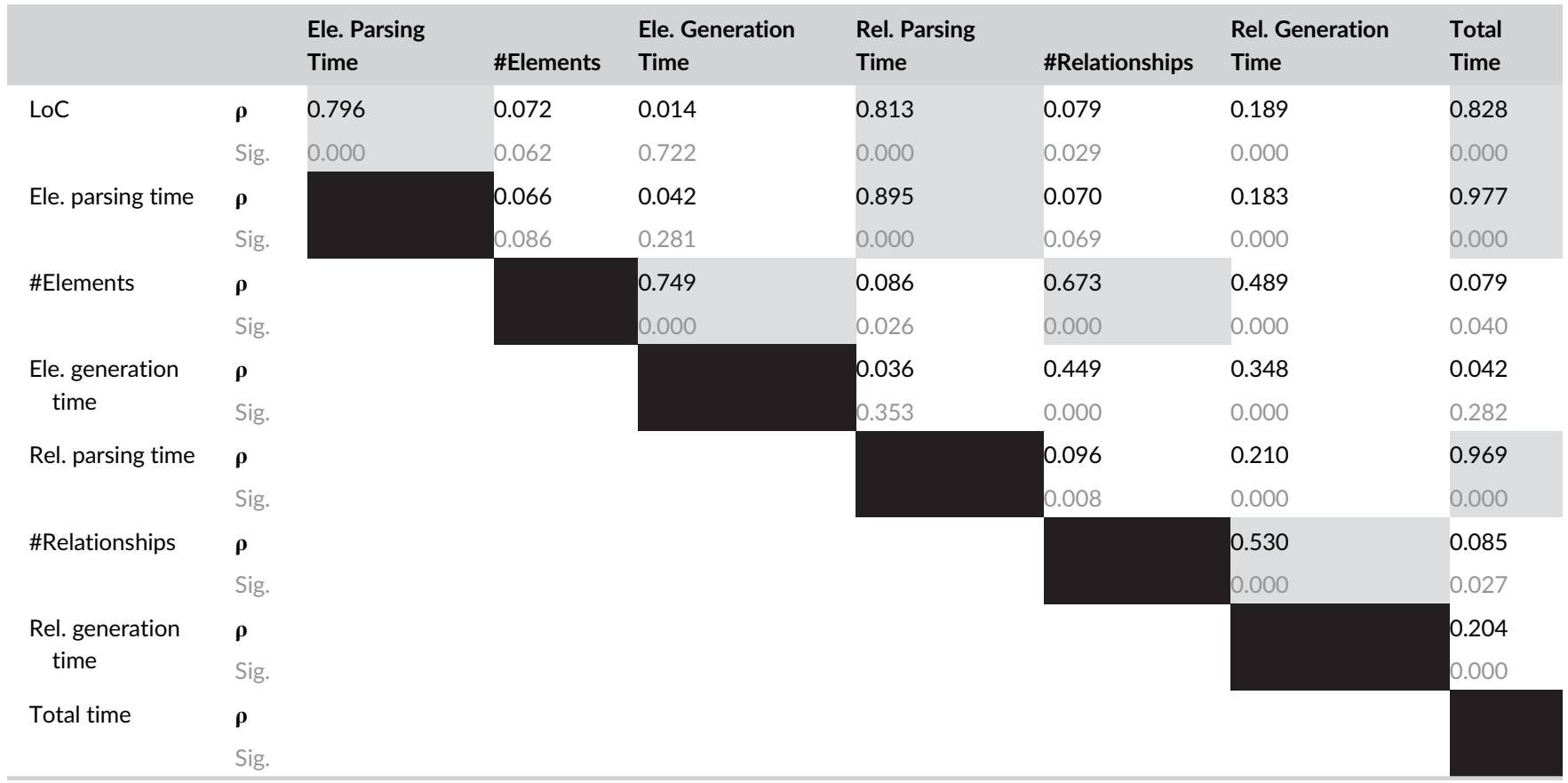



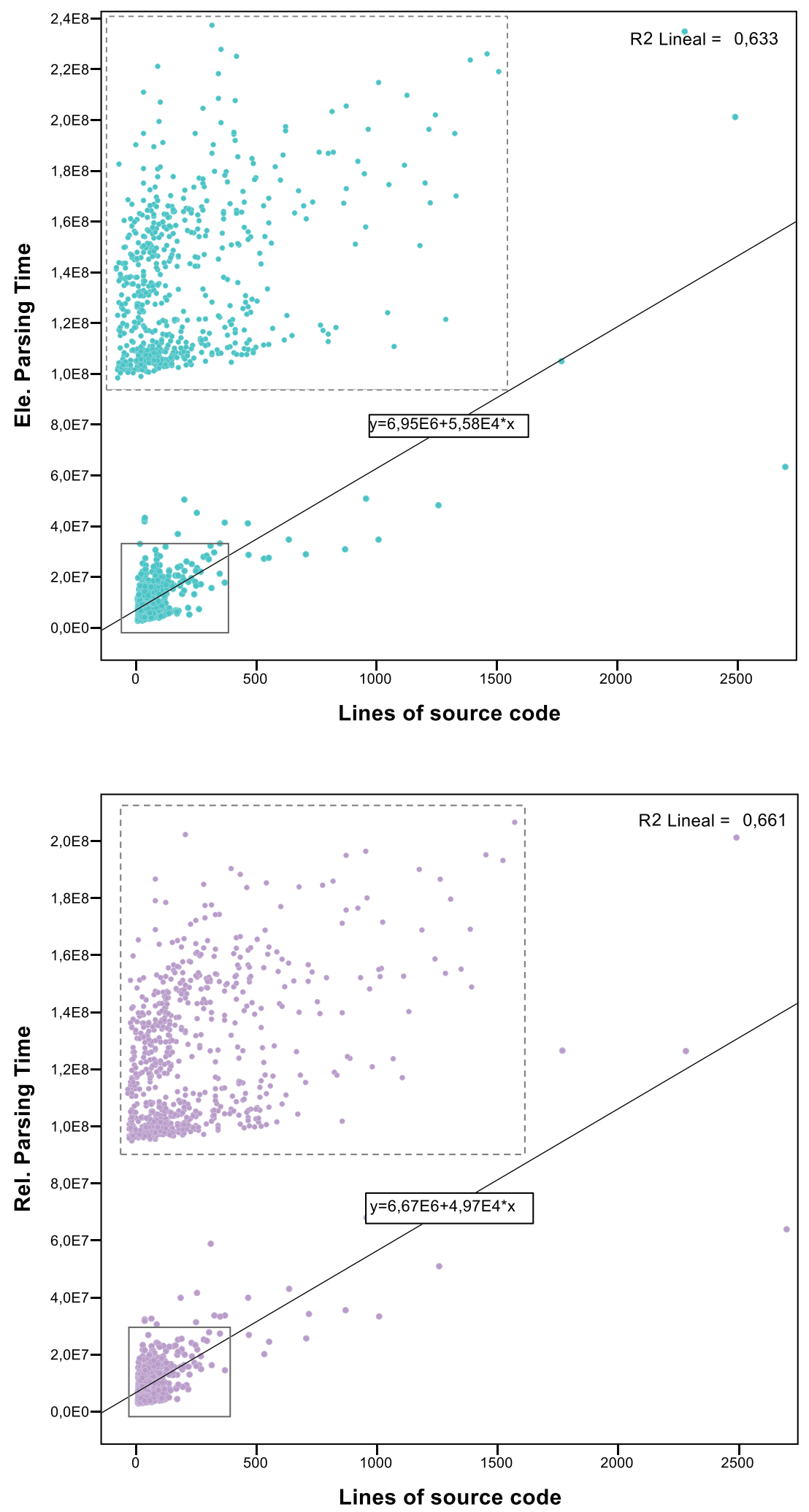

FIGURE 11 Scatter plot for ArchiMate elements parsing time and lines of source code
FIGURE 12 Scatter plot for ArchiMate relationships parsing time and lines of source code

these time measures have a linear correlation with the numbers of elements and relationships instead of the number of lines of source code, which makes more sense.

Having assessed the time spent on parsing source code and EA model generation, time spent on EA view generation is evaluated as well. It should be noticed that the parsing and EA model generation time is unique. Afterwards, all the selected views are generated in a row. A Pearson correlation test was performed for the total number of elements and relationships of the view (in this case the independent variable) and the 
generation time. The result was $\rho=0.987$ with a significance of 0.000040 . This shows a strong linear correlation between the number of selected ArchiMate items in the view and the time needed for achieving this.

Finally, Figure 13 shows a bar plot with the total time spent (in seconds) for generating every EA view. In total, the extraction process is around 20 seconds View generation takes between microseconds and $2 \mathrm{~s}$ for the case in which a view with all the elements of the models is generated. As a result, the time spent on view generation represents (in the worst case) $14 \%$ of the total time, while could be even neglectable for smaller views.

After analysing efficiency-related measures according to RQ2, all obtained insights demonstrated that the method under evaluation is scalable to bigger information systems because the time necessary for obtaining ArchiMate EA models and views is linearly correlated with the size of the system. As a result, there is no expected exponential growths in time.

\section{3 | Validity evaluation}

This section discusses threats to the validity to the case study and those possible actions to mitigate them. According to the literature, ${ }^{49,50}$ there are four main types of validity: internal, construct, external and reliability.

\subsection{1 | Construct validity}

This kind of validity refers to what extent the operational measures have a single interpretation and are valid to draw insights from them to answer the research questions stablished.

The provenance of precision and recall measures is the information retrieval field, where these metrics have demonstrated their validity. Actually, these measures have been used in many reverse engineering and mining techniques for assessing the effectiveness. These measures as computed based on experts' opinions allowed to determine whether the obtained ArchiMate model faithfully represent (or not) the organization's EA. Such opinions were collected from two experts, which can be different in some cases. So having an even number of experts is a threat to the construct validity. To mitigate this problem, experts tried to get an agreement when dissimilar opinions were expressed.

Together with those measures, size, connectivity and density as structural measures of the model have been used. These measures allowed to assess the understandability and modifiability of the EA models in a similar way it was previously addressed for business process models. $^{52,53}$

Moreover, several time-based measures were taken for evaluating the efficiency of the method under study. Because there are no benchmarks with which to compare these measures, it could entail a threat. To mitigate this, efficiency has been evaluated in term of the scalability. Thus, in order to assess the scalability of the method, some correlation tests were performed with these measures regarding size of the IS and its model.

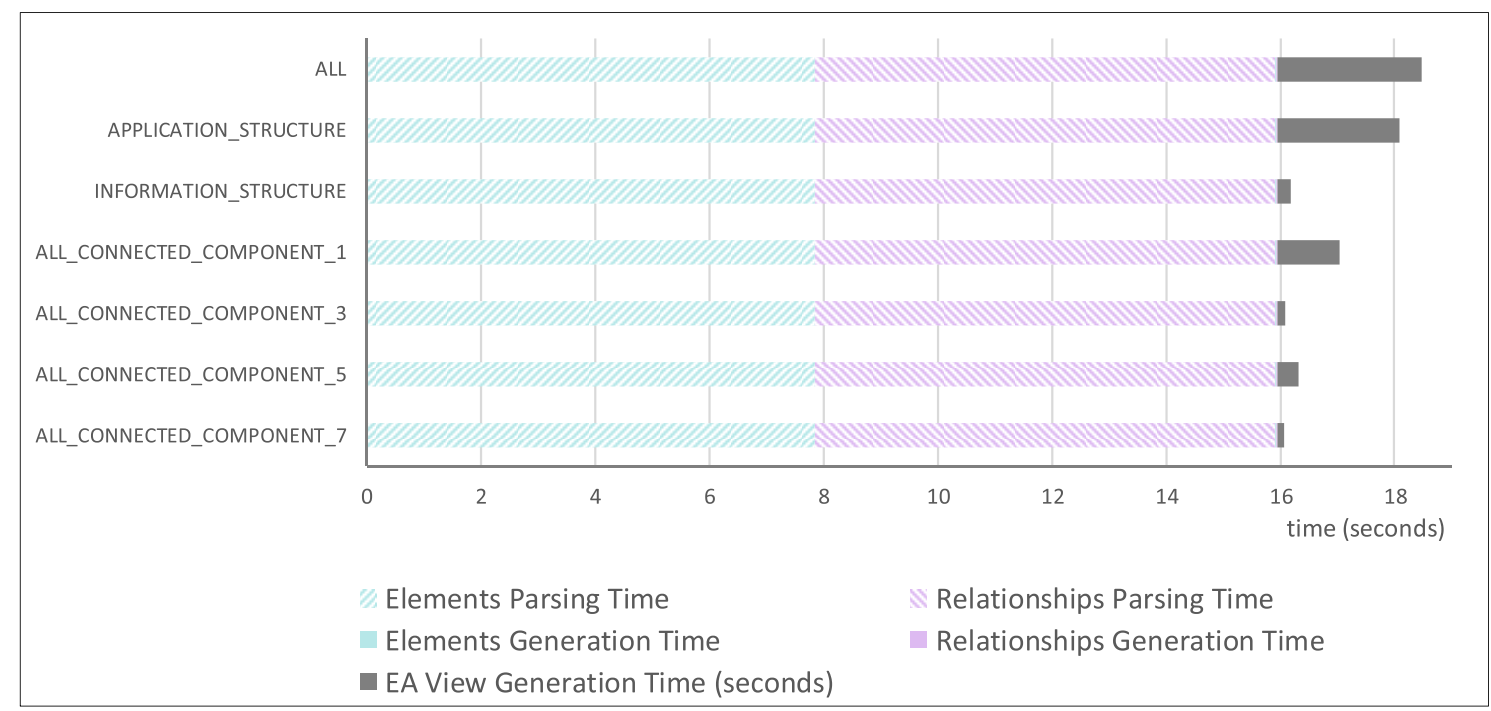

FIGURE 13 Bar plot for total time employed on generating ArchiMate EA views 


\subsection{2 | Internal validity}

There is no large population with regard to the analysis units, which were the EA views generated from a single EA model. Hence, results could be statistically less representative than expected. However, a clear trend for the proposed measures was identifiable in the case study. In the future, we hope to contrast the result of this case, by means of meta-analysis, with results obtained from further case studies considering another industrial IS. Furthermore, many viewpoints consider several EA models rather than a single one. As a result, next evolution of the ArchiRev tool will consider additional complex viewpoints in order to conduct case studies by generating other views.

Anyway, we can distinguish two main factors that could affect the results of this case study. First, the tool developed to support the ArchiRev method is a factor that could affect the models generated. As we mentioned, ArchiRev method could be instantiated according to three different dimensions (reverse engineering technique, the target EA viewpoint and the source artefacts). So the instantiation based on the static analysis of source code is a specific case of ArchiRev. Even so, the results could be different if a different implementation of the tool were used.

Second, the results for the precision and recall were computed after manual intervention, which is a factor that clearly threaten the internal validity. In that intervention, experts provided their subjective opinion about the obtained EA models/views. One possible mitigation might be to use an existing EA in the organization as a golden standard to be compared the generated one and, thus, compute precision and recall. Unfortunately, there was not an existing EA defined in the organization. Additionally, this threat could be mitigated through the comparison of automatically generated EA models with those being defined manually based on the knowledge of the experts. We guess that experts cannot remember every detail of the system; thus, the completeness of the outgoing EA might be poor. As a result, manually defined models can be considered as an appropriate golden standard. This is the reason why we decided to use a combined approach in which experts can model and fix the drafted EA model whereas they can rediscover some forgotten knowledge.

\subsection{3 | External validity}

This kind of validity is related to the degree of generalization of the obtained results. In other words, if the findings are relevant for other cases. Strictly, according to the tool used and the case selection criteria, the results obtained in this case study are only generalized to IS written in C\# that supports business operations. Also, as we mentioned in the internal validity section, results can be generalized to cases that consider static analysis of source code with the aim of generating application and information structure views. Anyway, further case studies are necessary to strengthen the generalization in a broader extent. As we mentioned, it was difficult to find companies encouraged to be involved in this kind of studies. Also, most of the requested companies were afraid about sharing their source code or, even worse, their customers' code. Moreover, EA models are usually associated with the strategical level of organizations, which prevent them to share any kind of information related to that critical making-decision level.

\subsection{4 | Reliability}

Finally, reliability concerns the extent in which data and the analysis are dependent of the researchers who conducted the study. A main threat to this is that the source code of the IS under study is not available because of disclosure agreements. To mitigate this, all the remaining information (including the generated EA model/views) is online available, ${ }^{55}$ in case other researchers want to check models or the dataset as well as used for replication or meta-analysis together other case studies. Also, the decisions made by experts about the EA elements generated through ArchiRev could differ if the selected experts are different. Anyway, these experts must be selected from the company that operates the information system, and they must know the business logic and EA concerns of the company.

\section{6 | CONCLUSIONS}

This paper proposes ArchiRev, a systematization work to automatically extract EA models, represented in ArchiMate, from various sources of knowledges. ArchiRev has been proposed as a generic and extensible method, which makes its application flexible. The ArchiRev method is defined as a cube with three dimensions. Thus, ArchiRev defines how various reverse engineering techniques together with IS-related artefacts (used as input) can be combined to generate different EA viewpoints.

The generic, extensible method has been tailored for analysing source code by means of static parser and thus extract valuable EA elements and its relationships in the application layer. Of course, these models obtained automatically might be combined with other parts of the EA defined manually by experts or the extracted models may even be refined by these experts. Despite that, automatic EA modelling contributes to reduce the error-proneness and time consumption of manual modelling and thus makes EA model realignment easier and faster. We believe that 
this proposal can help to combine EA with agile, maintaining a virtuous and necessary balance between rapid response to changes and alignment with enterprise goals. In other words, ArchiRev can be used as mean to achieve an effective BizDevOps. ${ }^{5,17}$

In order to demonstrate the suitability and applicability of the ArchiRev in an industrial context, the proposal has been applied to a reallife IS. Having generated the EA models from the source code of the IS, experts of the underlaying company analysed the model and its views to reach a verdict about precision, recall and F-measure of the retrieval process. The main insight was that the precision and recall are appropriate although recall was higher than precision, signifying that there were few missing elements, but various nonrelevant elements wrongly extracted. This means that manual postintervention by experts should be more focus on removing irrelevant elements instead of adding missing ones. This fact was more accentuated in the case of relationships between elements rather than in the own elements of the model. Regarding the efficiency analysis, the method demonstrated to be linear regarding the number of lines of source code. In particular, the inspection of source code was the most time-consuming part of the method (specially in comparison with the time devoted to EA model and view generation).

An important lesson learned during the case study has been the fact that technical expert intervention during the set-up of the extraction is critical. For example, the system under study was coded by using some framework that led to some technical, self-generated code that conditioned results of the analysis. For example, the use of specific framework can generate auxiliary code that then might be interpreted as false positive by the method. These false positive could be discarded by means of the definition of exclusions pattern as provided by the mentioned experts. In case technical experts could not be involved in the extraction process, a trial-error process approach might be used by using different configurations. This is possible because the process is automatic and scalable.

The future research of this work is currently focused on considering additional IS artefacts as well as reverse engineering technique to obtain richer and more accurate EA models with viewpoints at different abstraction layers. Having developed these further techniques framed in ArchiRev method, further case studies will be conducted for strengthening the obtained conclusions. Additionally, we are designing and implementing a simulator to understand in which extend an EA viewpoint can be achieved with specific reverse engineering techniques and the use of specific input artefacts and experts as sources of knowledge. Results of this research will offer a better understanding of relationships provided in Table 1. Finally, we are investigating how ArchiRev could be effectively used in broader contexts like BizDevOps. We will define additional usage scenarios for ArchiRev to help companies to continuously redefine business goals and processes and the respective functionality of their services and applications. Thus, ArchiRev might aid companies to (re)develop and operating such applications in a more agile way but also in a more suitable manner regarding business goals.

\section{ACKNOWLEDGEMENTS}

This study has been partially funded by the following projects: G3SOFT (SBPLY/17/180501/000150), GEMA (SBPLY/17/180501/000293) and SOS (SBPLY/17/180501/000364), funded by the "Dirección General de Universidades, Investigación e Innovación-Consejería de Educación, Cultura y Deportes and Gobierno de Castilla-La Mancha (JCCM)." This work is also part of the projects BIZDEVOPS-Global (RTI2018-098309-B-C31) and ECLIPSE (RTI2018-094283-B-C31) funded by Ministerio de Economía, Industría y Competitividad y Fondos FEDER.

\section{ORCID}

Ricardo Pérez-Castillo (D) https://orcid.org/0000-0002-9271-3184

Danilo Caivano (iD) https://orcid.org/0000-0001-5719-7447

Francisco Ruiz (D) https://orcid.org/0000-0002-4923-7848

Mario Piattini (D) https://orcid.org/0000-0002-7212-8279

\section{REFERENCES}

1. Ebert C, Duarte CHC. Digital transformation. IEEE Softw. 2018;35(4):16-21. https://doi.org/10.1109/MS.2018.2801537

2. Silva NMCD, Silva MLBMD, Sousa PMVAD. A viewpoint for analyzing enterprise architecture evolution. In 2017 IEEE 21 st International Enterprise Distributed Object Computing Conference (EDOC). 2017:20-29. https://doi.org/10.1109/edoc.2017.13

3. Zimmermann A, Schmidt R, Sandkuhl K, Wissotzki M, Jugel D, Mohring M. Digital enterprise architecture-transformation for the internet of things. In 2015 IEEE 19th International Enterprise Distributed Object Computing Workshop. 2015: Adelaide, SA, Australia; 130-138. https://doi.org/10.1109/ edocw.2015.16

4. Putano B. A quick guide to BizDevOps. 2017 17/11/2017 [cited 2019 11/03/2019]; Available from: https://stackify.com/bizdevops-guide/

5. Drews P, Schirmer I, Horlach B, Tekaat C. Bimodal enterprise architecture management: the emergence of a new EAM function for a BizDevOps-Based Fast IT. In 2017 IEEE 21st International Enterprise Distributed Object Computing Workshop (EDOCW). 2017. https://doi.org/10. 1109/EDOCW.2017.18

6. Kotusev S, Singh M, Storey I. Consolidating enterprise architecture management research. In 2015 48th Hawaii International Conference on System Sciences. 2015:4069-4078. https://doi.org/10.1109/hicss.2015.489

7. Veneberg RKM, lacob ME, van Sinderen MJ, Bodenstaff L. Relating business intelligence and enterprise architecture-a method for combining operational data with architectural metadata. Int J Coop Inf Syst. 2016;25(02):1650007. https://doi.org/10.1142/s0218843016500076 
8. Chen D, Doumeingts G, Vernadat F. Architectures for enterprise integration and interoperability: past, present and future. Comput Ind. 2008;59(7): 647-659. https://doi.org/10.1016/j.compind.2007.12.016

9. Scholtz B, Calitz A, Connolley A. An analysis of the adoption and usage of enterprise architecture. In Proceedings of the First International Conference on Enterprise Systems: ES 2013. 2013: Cape Town, South Africa;1-9. https://doi.org/10.1109/es.2013.6690087

10. Op't Land M, Proper E, Waage M, Cloo J, Steghuis C. In: T.E.E. Series, ed. Enterprise architecture: creating value by informed governance. The Enterprise Engineering Series. Heidelberg: Springer; 2009 n.p.

11. Tribolet J, Sousa P, Caetano A. The role of enterprise governance and cartography in enterprise engineering. Enterp Model Inf Syst Archit (EMISAJ). 2014;9(1):38-49.

12. Farwick M, Pasquazzo W, Breu R, Schweda CM, Voges K, Hanschke I. A meta-model for automated enterprise architecture model maintenance. In 2012 IEEE 16th International Enterprise Distributed Object Computing Conference; 2012:1-10. https://doi.org/10.1109/edoc.2012.11

13. Kulkarni V, Clark T, Barat S, Barn B. Model based enterprise simulation and analysis. A pragmatic approach reducing the burden on experts. In International Conference on Conceptual Modeling (ER 2014). Springer Verlag; 2014:3-12.

14. The Open Group. The ArchiMate 3.0 Enterprise Architecture Modeling Language. 2016. http://www.opengroup.org/subjectareas/enterprise/ archimate-overview

15. Sánchez M, Reyes JC, Villalobos J. Extraction and reconstruction of enterprise models. In Lecture Notes in Business Information Processing. Springer Verlag; 2014:3-20. https://doi.org/10.1007/978-3-662-44860-1_1

16. Loucopoulos P, Kavakli E. Capability oriented enterprise knowledge modeling: the CODEK approach. In: Domain-Specific Conceptual Modeling. Springer International Publishing; 2016:197-215. https://doi.org/10.1007/978-3-319-39417-6_9

17. Gruhn V, Schäfer C. BizDevOps: because DevOps is not the end of the story. In Communications in Computer and Information Science. 2015: 388-398. https://doi.org/10.1007/978-3-319-22689-7_30

18. Kaisler SH, Armour F, Valivullah M. Enterprise architecting: critical problems. In System Sciences, 2005. HICSS'05. Proceedings of the 38th Annual Hawaii International Conference on, IEEE: Big Island, HI, USA, USA; 2005:224b-224b.

19. Bente S, Bombosch U, Langade S. Toward pragmatism. In: collaborative enterprise architecture. Boston: Morgan Kaufmann; 2012:159-234. https://doi. org/10.1016/b978-0-12-415934-1.00007-8

20. Farwick M. Towards automation of enterprise architecture model maintenance. In 24th International Conference on Advanced Information Systems Engineering (CAiSE'12) - Doctoral Consortium, I. Mirbel and B. Pernici, Editors. Gdansk, Poland; 2012:1-11.

21. Pérez-Castillo R, Ruiz F, Piattini M. A decision-making support system for enterprise architecture modelling. Decis Support Syst. 2020;131:113249. https://doi.org/10.1016/j.dss.2020.113249

22. Bogner J, Zimmermann A. Towards integrating microservices with adaptable enterprise architecture. In 2016 IEEE 20 th International Enterprise Distributed Object Computing Workshop (EDOCW); 2016. https://doi.org/10.1109/EDOCW.2016.7584392

23. Farwick M, Breu R, Hauder M, Roth S, Matthes F. Enterprise architecture documentation: empirical analysis of information sources for automation. In 2013 46th Hawaii International Conference on System Sciences; 2013. https://doi.org/10.1109/HICSS.2013.200

24. Hilliard R, Malavolta I, Muccini H, Pelliccione P. On the composition and reuse of viewpoints across architecture frameworks. In 2012 Joint Working IEEE/IFIP Conference on Software Architecture and European Conference on Software Architecture; 2012. https://doi.org/10.1109/WICSA-ECSA. 212.21

25. Repta D, Moisescu MA, Sacala IS, Stanescu AM, Neagu G. Automated process mapping for cyber intelligent enterprise. In 201520 th International Conference on Control Systems and Computer Science; 2015. https://doi.org/10.1109/CSCS.2015.126

26. Perez-Castillo R, Ruiz-Gonzalez F, Genero M, Piattini M. A systematic mapping study on enterprise architecture mining. Enterp Inf Syst. 2019;13(5): 675-718. https://doi.org/10.1080/17517575.2019.1590859

27. Ducasse S, Pollet D. Software architecture reconstruction: a process-oriented taxonomy. IEEE Trans Softw Eng. 2009;35(4):573-591. https://doi.org/ 10.1109/TSE.2009.19

28. Kim H, Oussena S, Essien J, Komisarczuk P. Towards event-driven enterprise architecture. In: Uncovering Essential Software Artifacts through Business Process Archeology. IGI Global; 2014:285-311. https://doi.org/10.4018/978-1-4666-4667-4.ch011

29. Clark T, Barn BS, Oussena S. LEAP: a precise lightweight framework for enterprise architecture. In 4th India Software Engineering Conference. Thiruvananthapuram, Kerala, India; 2011:85-94. https://doi.org/10.1145/1953355.1953366

30. Alwadain A, Fielt E, Korthaus A, Rosemann M. Empirical insights into the development of a service-oriented enterprise architecture. Data Knowl Eng. 2016;105:39-52. https://doi.org/10.1016/j.datak.2015.09.004

31. ISO/IEC/IEEE. Systems and software engineering-architecture description. ISO/IEC/IEEE 42010:2011(E) (Revision of ISO/IEC 42010:2007 and IEEE Std 1471-2000), 2011:1-46. https://doi.org/10.1109/ieeestd.2011.6129467

32. The Open Group. TOGAF. Enterprise Edition. Version 9.1. 2011. http://www.togaf.org

33. The Open Group. TOGAF ${ }^{\circledR}$ Worldwide. 2016 [cited 2018; Available from: http://www.opengroup.org/subjectareas/enterprise/togaf/worldwide

34. Simon D, Fischbach K, Schoder D. An exploration of enterprise architecture research. Commun Assoc Inf Syst. 2013;32(1):1-72. https://aisel.aisnet. $\mathrm{org} / \mathrm{cgi} / \mathrm{view}$ content.cgi?article=3684\&context=cais

35. van der Aalst WMP. Process Mining: Discovery, Conformance and Enhancement of Business Processes. Berlin, Heidelberg: Springer; 2011. https://doi.org/ 10.1007/978-3-642-19345-3

36. Van Langerak R, Van Der Werf JMEM, Brinkkemper S. Uncovering the runtime enterprise architecture of a large distributed organisation. In: $A$ process mining-oriented approach. Springer Verlag; 2017:247-263. https://doi.org/10.1007/978-3-319-59536-8_16

37. Liu C, van Dongen BF, Assy N, van der Aalst WM. A general framework to identify software components from execution data. In 14 th International Conference on Evaluation of Novel Approaches to Software Engineering (ENASE'19). INSTIC: Heraklion, Crete, Greece; 2019:234-241.

38. Truong TM, Lê LS, Tôn LP. Re-engineering enterprises using data warehouse as a driver and requirements as an enabler. In 2017 IEEE 21 st International Enterprise Distributed Object Computing Conference (EDOC). 2017. https://doi.org/10.1109/EDOC.2017.18

39. van der Werf JMEM, van Schuppen C, Brinkkemper S, Jansen S, Boon P, Plas Gvd. Architectural intelligence: a framework and application to e-learning. In RADAR+EMISA@CAiSE. 2017.

40. Ge B, Hipel KW, Yang K, Chen Y. A novel executable modeling approach for system-of-systems architecture. IEEE Syst J. 2014;8(1):4-13. https://doi. org/10.1109/JSYST.2013.2270573 
41. Hu J, Huang L, Chang X, Cao B. A model driven service engineering approach to system of systems. In 2014 IEEE International Systems Conference Proceedings. 2014. https://doi.org/10.1109/SysCon.2014.6819248

42. Granchelli G, Cardarelli M, Francesco PD, Malavolta I, lovino L, Salle AD. Towards recovering the software architecture of microservice-based systems. In 2017 IEEE International Conference on Software Architecture Workshops (ICSAW). 2017. https://doi.org/10.1109/ICSAW.2017.48

43. Santana A, Simon D, Fischbach K, Moura HD. Combining network measures and expert knowledge to analyze enterprise architecture at the component level. In 2016 IEEE 20th International Enterprise Distributed Object Computing Conference (EDOC). 2016. https://doi.org/10.1109/ EDOC.2016.7579379

44. Pulkkinen M, Kapraali L. Collaborative EA information elicitation method: the IEM for business architecture. In 2015 IEEE 17 th Conference on Business Informatics. Lisbon, Portugal; 2015:64-71. https://doi.org/10.1109/CBI.2015.33

45. Rosasco N, Dehlinger J. A case study investigation of a lightweight, systematic elicitation approach for enterprise architecture requirements. Springer Verlag; 2015:33-45. https://doi.org/10.1007/978-3-319-27218-4_3

46. Lau K-K, Arshad R. A concise classification of reverse engineering approaches for software product lines. International Journal On Advances in Software: ICSEA 2016: The Eleventh International Conference on Software Engineering Advances, 2016.

47. The Open Group. Enterprise continuum. TOGAF ${ }^{\circledR} 9.1$ 2011; Available from: http://pubs.opengroup.org/architecture/togaf91-doc/arch/chap39.html

48. Buckl S, Matthes F, Neubert C, Schweda CM. A Lightweight Approach to Enterprise Architecture Modeling and Documentation. Berlin, Heidelberg: Springer Berlin Heidelberg; 2011.

49. Runeson P, Host M, Rainer A, Regnell B. Case Study Research in Software Engineering: Guidelines and Examples. Hoboken, USA: John Wiley \& Sons; 2012 n.p.

50. Yin RK. Case Study Research. Design and Methods. 5th ed. London: SAGE Publications. n.p.; 2013:312.

51. Pérez-Castillo R, de Guzmán IG-R, Piattini M. Business process archeology using MARBLE. Inform Softw Technol. 2011;53(10):1023-1044. https://doi. org/10.1016/j.infsof.2011.05.006

52. Sánchez-González L, Rubio FG, Mendling J, González FR. Quality assessment of business process models based on thresholds. In: On the Move to Meaningful Internet Systems. Vol. 2010. Hersonissos, Crete, Greece: OTM; 2010:78-95.

53. Mendling J, Reijers HA, and Cardoso J. What makes process models understandable?, In International Conference on Business Process Management (BPM 2007). Springer;2007:48-63. https://doi.org/10.1007/978-3-540-75183-0_4

54. Pérez-Castillo R, Fernández-Ropero M, Piattini M. Business process model refactoring applying IBUPROFEN. An industrial evaluation. J Syst Softw. 2019;147:86-103. https://doi.org/10.1016/j.jss.2018.10.012

55. Pérez-Castillo R. Extra material of ArchiRev-reverse engineering of information systems toward archimate models. An Industrial Case Study. 2018 [cited 2018 22/12/2018]; Available from: https://alarcos.esi.uclm.es/per/rpdelcastillo/ex_ea_archirev.html

56. Rowe G, Wright G. The Delphi technique as a forecasting tool: issues and analysis. Int J Forecast. 1999;15(4):353-375.

How to cite this article: Pérez-Castillo R, Caivano D, Ruiz F, Piattini M. ArchiRev-Reverse engineering of information systems toward ArchiMate models. An industrial case study. J Softw Evol Proc. 2020;e2314. https://doi.org/10.1002/smr.2314 